\title{
Ethnomedicinal Use, Phytochemistry, and Pharmacology of Xylocarpus granatum J. Koenig
}

\author{
Dipta Dey, ${ }^{1}$ Cristina Quispe, ${ }^{2}$ Rajib Hossain, ${ }^{3}$ Divya Jain, ${ }^{4}$ Rasel Ahmed Khan, ${ }^{5}$ \\ Pracheta Janmeda, ${ }^{4}$ Muhammad Torequl Islam (D), ${ }^{3}$ Hafiz Ansar Rasul Suleria (D), \\ Miquel Martorell $\mathbb{D}^{7,8}$ Sevgi Durna Daştan, ${ }^{9,10}$ Manoj Kumar $\mathbb{D}^{11}$ ' ${ }^{11}$ asaman Taheri $\left(\mathbb{D},{ }^{12}\right.$ \\ Anka Trajkovska Petkoska, ${ }^{13}$ and Javad Sharifi-Rad (iD) \\ ${ }^{1}$ Department of Biochemistry and Molecular Biology, Life Science Faculty, \\ Bangabandhu Sheikh Mujibur Rahman Science and Technology University, Gopalganj 8100, Bangladesh \\ ${ }^{2}$ Facultad de Ciencias de la Salud, Universidad Arturo Prat, Avda. Arturo Prat 2120, Iquique 1110939, Chile \\ ${ }^{3}$ Department of Pharmacy, Life Science Faculty, Bangabandhu Sheikh Mujibur Rahman Science and Technology University, \\ Gopalganj 8100, Bangladesh \\ ${ }^{4}$ Department of Bioscience and Biotechnology, Banasthali Vidyapith, Vanasthali, India \\ ${ }^{5}$ Pharmacy Discipline, Khulna University, Khulna, Bangladesh \\ ${ }^{6}$ Department of Agriculture and Food Systems, The University of Melbourne, Melbourne 3010, Australia \\ ${ }^{7}$ Department of Nutrition and Dietetics, Faculty of Pharmacy, and Centre for Healthy Living, University of Concepción, \\ Concepción 4070386, Chile \\ ${ }^{8}$ Universidad de Concepción, Unidad de Desarrollo Tecnológico, UDT, Concepción 4070386, Chile \\ ${ }^{9}$ Department of Biology, Faculty of Science, Sivas Cumhuriyet University, Sivas 58140, Turkey \\ ${ }^{10}$ Beekeeping Development Application and Research Center, Sivas Cumhuriyet University, Sivas 58140, Turkey \\ ${ }^{11}$ Chemical and Biochemical Processing Division, ICAR-Central Institute for Research on Cotton Technology, \\ Mumbai 400019, India \\ ${ }^{12}$ Phytochemistry Research Center, Shahid Beheshti University of Medical Sciences, Tehran, Iran \\ ${ }^{13}$ Faculty of Technology and Technical Sciences, St. Kliment Ohridski University, Bitola 1400, North Macedonia
}

Correspondence should be addressed to Muhammad Torequl Islam; dmt.islam@bsmrstu.edu.bd, Miquel Martorell; mmartorell@ udec.cl, and Javad Sharifi-Rad; javad.sharifirad@gmail.com

Received 19 May 2021; Revised 7 August 2021; Accepted 10 August 2021; Published 31 August 2021

Academic Editor: Talha Bin Emran

Copyright (C) 2021 Dipta Dey et al. This is an open access article distributed under the Creative Commons Attribution License, which permits unrestricted use, distribution, and reproduction in any medium, provided the original work is properly cited.

The mangrove plants are the potential sources of foods and remedies for people living in the forests and nearby communities. Xylocarpus granatum J. Koenig is traditionally used to treat various diseases including diarrhea, cholera, dysentery, fever, malaria, and viral infections, among others. To summarize critically the taxonomy, ethnomedicinal, phytochemistry, and pharmacological activities of X. granatum, information was collected from different databases. An up-to-date search (till June 2020) was carried out with the help of various scientific web resources from databases such as PubMed, Science Direct, Google Scholar, and various patent offices (e.g., WIPO, CIPO, and USPTO) using the keywords "Xylocarpus granatum" and then paired with ethnomedicinal use and phytochemical, phytochemistry, and pharmacological activity (in vitro, ex vivo, and in vivo studies). Findings revealed that seeds, fruits, stem bark, leaf, and twigs of $X$. granatum exhibited a wide range of key phytochemicals including limonoids, phragmalin, limonoid-based alkaloids, mexicanolides, protolimonoids, flavonols, and lactones. The plant possessed potent antioxidant, anticancer, antidiabetic, antimicrobial, antimalarial, antifeedant, and neuroprotective activities. No clinical studies have been reported in the databases. Ethnomedicinal assessment indicated the application of X. granatum in various fields of medical science specially to treat various human ailments, and this was attributed to the presence of enormous alkaloids as confirmed by pharmacological studies. However, to understand the mechanism of action in-depth studies are required. In view of these findings, more research is necessary to explore and characterize the chemical compounds and toxicological aspects of this medicinal mangrove plant. Overall, it can be stated that $X$. granatum may be one of the hopeful medicinal herbs for the treatment of various diseases in human beings. 


\section{Introduction}

Traditional medicines like Chinese traditional medicine, Ayurveda, Unani, and Korean traditional medicine have been used extensively ever since the ancient times. These medicines are based on using natural products for treating various human ailments [1]. Use of new medicines with no doubt helps treat different diseases, but it also poses a risk of side effects. Increased demands for reducing these side effects of the current drugs are driving researchers and pharmacologist towards formulating natural plant-based drugs, thereby exploring different traditionally used medicinal plants/herbs [1]. Xylocarpus granatum J. Koenig is a good example of a medicative plant that is used as a traditional herbal drug [2]. $X$. granatum is a species of the mahogany family and is widely distributed in the coastal forests of Bengal, Burma, Malay Peninsula, Andaman, and Africa. In Bangladesh, this plant is found in low lying, a swampy locality in the Sundarbans mangrove forest [3]. It is spread across the coastal areas of tropical and subtropical zones and river delta. Typically, this plant's different parts are used for different purposes, most popularly used for the treatment of diarrhea, dyslipidemia, fever, inflammation, malaria, cholera, dysentery, and so on $[4,5]$. Various types of chemical compounds are extracted from the different parts of the plant such as limonoids, phragmalin, limonoids based alkaloids, mexicanolides, protolimonoids, flavonol-like compound, lactone, ethanol extract, methanol extract, and alkaloids, among others [6, 7]. Limonoids are the most vital chemical component that is isolated from the different parts of the $X$. granatum plant [8]. Numerous limonoids are isolated from different parts of $X$. granatum such as fruit peel, seed kernels, seed, fruit, and seed coat [9]. Limonoids possess diverse pharmacological activities, such as antimicrobial, antimalarial, antiviral, anticancer, antidiabetic, antioxidant, antifeedant [10], and neuroprotective effects [11]. Other potential activities $X$. granatum extracts include antifilarial, antidepressant, antimalarial [12], and antisecretory effects [13]. So, the limonoids are important pharmacologically active constituents of $X$. granatum fruit that need to be exploited further [9]. A more detailed investigation need to be done on the toxicological effects and clinical trials on humans.

This review offers up-to-date information on the phytochemical profile and biopharmacological effects of $X$. granatum on the basis of scientific reports found in the databases.

\section{Research Methodology}

The literature on X. granatum botanical description, ethnomedicinal uses, secondary metabolites, and biological properties was collected, analyzed, and summarized in this review. Scientific search engines, such as PubMed, ScienceDirect, SpringerLink, Web of Science, Scopus, Wiley
Online, SciFinder, and Google Scholar, and various patent offices (e.g., WIPO, CIPO, and USPTO), were used to collect all published articles about this species. Several terms were used as keywords like Xylocarpus granatum, chemical compounds and Xylocarpus granatum, taxonomical classification, antidiabetic and Xylocarpus granatum, and limonoids and their pharmacological activity. All published work on X. granatum in different languages (English) was cited in this study. The identification and examination of the collected manuscripts were based on their titles and abstracts. Reference lists of the retrieved papers were also examined to identify further relevant papers. Chemical structures were drawn using ChemSketch version 12.01 software.

\section{Result and Discussion}

3.1. Botanical Description. The word Xylocarpus means woody fruit (in Latin) and refers to the large and distinctly woody fruit and seeds of this genus. X. granatum taxonomical classification is given as follows [14]. The maxi-

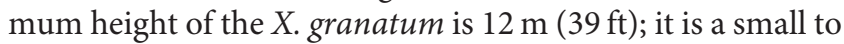
medium-sized evergreen tree. The evergreen leaves of the tree have special characteristics; the leaves are pinnate and arranged spirally on the twigs (Figure 1). The trunk has buttresses and above-ground roots, which extend for long distances to either side. $X$. granatum flowers are white or pinkish-yellow; each flower is $8 \mathrm{~mm}$ wide with four parts. The flowers are produced spherical, large, woody capsules, $9-12 \mathrm{~cm}$ in diameter. If the capsules are split, this brings out a dozen seeds.

3.2. Taxonomy and Geographic Distribution. The taxonomical classification of X. granatum [14] is as follows:

Kingdom: Plantae

Phylum: Magnoliophyta

Class: Eudicots

Order: Sapindales

Family: Meliaceae

Genus: Xylocarpus

Species: Xylocarpus granatum J. Koenig

$X$. granatum, a species of mangrove in the mahogany family (Meliaceae), is commonly known as "cannonball mangrove, cedar mangrove [15], dhundul [16], or puzzle nut tree" [17]. Asia, Africa, Australia [18], and the Pacific Islands are the main inhabitant of this plant [15]. This plant is found in low lying, swampy locality in the Sundarbans mangrove forests in Bangladesh [3]. This forest covers $6017 \mathrm{~km}^{2}$ in Bangladesh and mangroves are salt-tolerant forest ecosystems of tropical and subtropical intertidal regions of the world [3]. This plant extends from Tanzania, Kenya, and Mozambique to Malaysia, Indonesia, Thailand, Papua New Guinea, India, Bangladesh, and northern Australia [3]. This species is native to the tropical and subtropical western Indo-Pacific region [19]. 


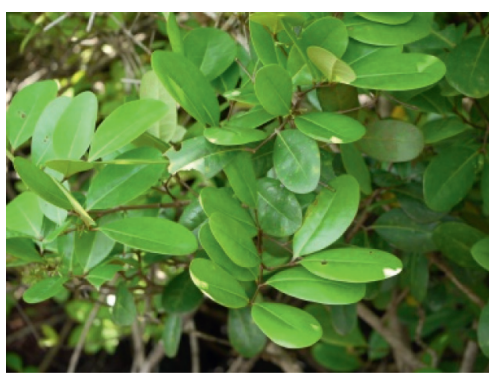

(a)

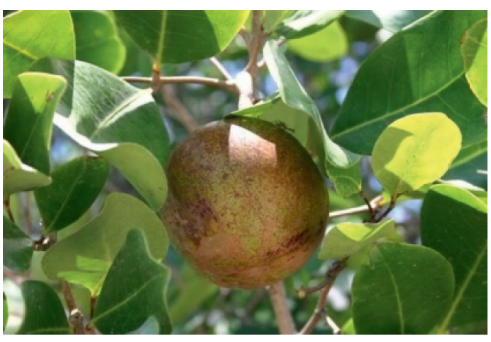

(d)

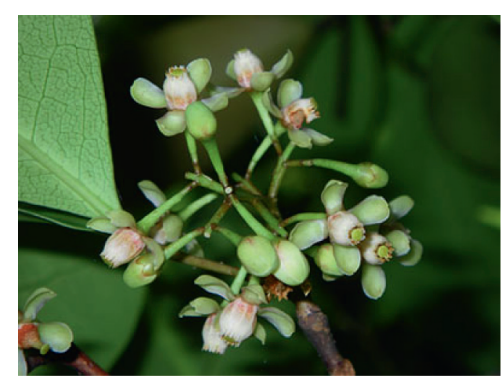

(b)

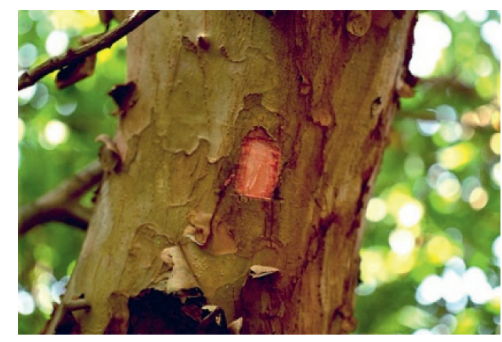

(e)

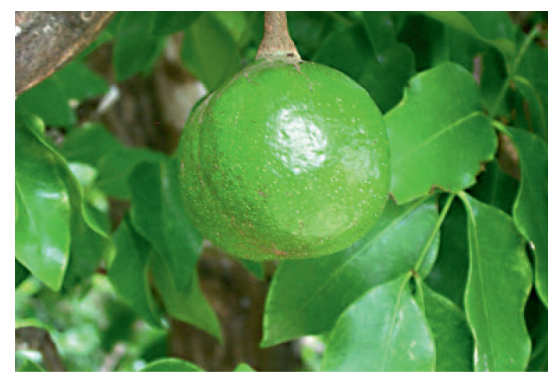

(c)

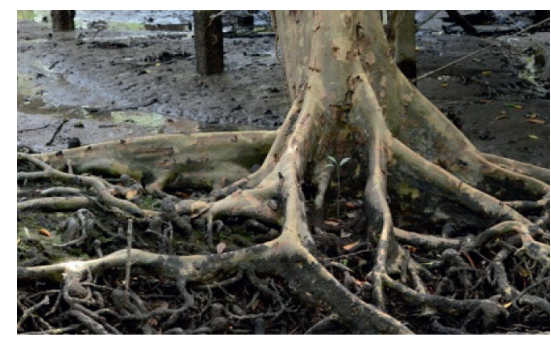

(f)

Figure 1: Different parts of Xylocarpus granatum J Koenig. (a) Leaves, (b) flowers, (c) unripe fruit, (d) ripe fruit, (e) stem and bark, and (f) roots.

3.3. Ethnopharmacological Uses. X. granatum, a puzzle nut tree, is a mangrove species belonging to mahogany family $[20,21]$. It is commonly known as "pussur" in Hindi and "dhundul" in Bengali. It is an evergreen tree having moderate-sized grey bark, usually growing in coastal forests of Bengal, Burma, the Malaya peninsula, Andaman Islands, and island of Australia and Africa [12]. It is a medicinal plant and used by different ethnic communities all over the world [22]. Several reports suggested that from past to present this plant has significant medicinal properties [23]. X. granatum plants have several bioactive compounds, including gedunin and limonoids [6, 24]. All of these compounds possess significant inhibitory activity against cancer $[16,25]$ and malarial, viral, feedant, diabetes, filarial activity, and fungicidal activity from ancient times $[10,26]$. All of these compounds extracted from the twigs and leaves, fruits, seeds, barks, seed kernels, and stem bark.

In the previous studies, researchers present various traditional uses of $X$. granatum extract. In southeast Asia, it is used as a treatment of diarrhea, fevers such as malaria, viral diseases like influenza, and cholera and also as an antifeedant or insecticide throughout ancient times $[8,25]$. In folklore, the $X$. granatum plant has been applied to treat malaria, fever, cholera, dysentery, and diarrhea in many countries including India. The extraction of leaves, fruits, and barks of $X$. granatum possesses potential free radical scavenging activities [4]. Besides, Uddin et al. [16] proposed that this plant extract chemicals have a variety of medicinal properties and in Bangladesh, and it was employed for the treatment of fever, malaria, cholera, diarrhea, and dysentery. Also, antioxidant and antifilarial activities have been reported from ancient times [16].
3.4. Phytochemistry. The $X$. granatum plant contains several bioactive constituents (Table 1 and Figure 2). Limonoids are the most vital chemical component that are isolated from the different parts of the X. granatum plant [8]. Moreover, $X$. granatum's seeds consist of sundarbanxylogranins A-E [27]; krishnagranatins A-I [28]; thaixylogranins A-H [29]; granaxylocarpins A-E [30]; xylocarpanoids A and B [31]; xylomexicanins A-D, I, and J [32-34]; xyloccensins I, K, L, $\mathrm{O}-\mathrm{S}, \mathrm{V}, \mathrm{W}$, and $\mathrm{Y}[10,12,35,36]$; hainangranatumins $\mathrm{A}-\mathrm{J}$ [37]; xylogranatins F-R [38]; thaigranatins A-E [39]; xylocartin C [40]; andhraxylocarpins A-E [41]; protoxylogranatin A-B [42, 43]; protoxylocarpins F-H [25].

The plant fruit contains gedunin $[16,40]$, andirobin, mexicanolide, and phragmalin [40]; cipadesin A [2]; xylocarpins A-I [8]; photogedunin [48]; xylocarpin L [35]; xyloccensin I, Y, X1, X2 [49]; xylogranatin E [50]; xylogranatinin [51]. Furthermore, stem bark of $X$. granatum consists of xyloccensin $\mathrm{M}$ and $\mathrm{N}$ [44]; xyloccensins Q-U [10]; xyloccensin L [45]; xyloccensin I and J [46]; xyloccensins $\mathrm{O}$ and $\mathrm{P}$ [47]. Leaf and twigs contain lactone [22]; xyloccensins $\mathrm{O}-\mathrm{S}$ and $\mathrm{V}$ [36]; xylogranin $\mathrm{B}$ [26]; xylogranatopyridines A and B [52]; xylogranatumines A-G [53]; xylogranatin E [50], and root bark contains N-methylflindersine [54].

It is evident that $X$. granatum is rich in various compounds, but limonoids, which is an oxidized tetranortriterpenoid derivative, is most dominant and widely studied secondary metabolite. This group of compounds is gaining more and more interest of scientific community due to its various health promoting effects. There are as many as 100 limonoids that are extracted and characterized. Limonoids have important biological activities such as anticancer, antioxidant, antiulcer, antimicrobial, and antiviral actions [55]. Traditionally, these mangroves are also 
TABle 1: Phytochemistry of Xylocarpus granatum J. Koenig.

\begin{tabular}{|c|c|c|}
\hline Parts & Compounds & References \\
\hline Seeds & $\begin{array}{l}\text { Sundarbanxylogranins A-E; krishnagranatins A-I; thaixylogranins A-H; granaxylocarpins A-E; } \\
\text { xylocarpanoids A and B; xylomexicanins A-D, I, and J; xyloccensin I, K, L, O-S, V, W, and Y; } \\
\text { hainangranatumins A-J; xylogranatins F-R; thaigranatins A-E; xylocartin C; andhraxylocarpins } \\
\text { A-E; protoxylogranatin A-B; protoxylocarpins F-H }\end{array}$ & {$[10,12,25,27-43]$} \\
\hline Stem bark & Xyloccensin M and N; xyloccensins Q-U; xyloccensin L; xyloccensin I, J; xyloccensins $\mathrm{O}$ and P & $-47]$ \\
\hline Fruits & $\begin{array}{l}\text { Gedunin; andirobin; mexicanolide; phragmalin; cipadesin A; xylocarpins A-I; photogedunin, } \\
\text { xylocarpin L, xyloccensin I, Y, X1, and X2; xylogranatin E; xylogranatinin }\end{array}$ & {$[2,8,16,35,40,48-51]$} \\
\hline $\begin{array}{l}\text { Leaf and } \\
\text { twigs }\end{array}$ & $\begin{array}{c}\text { Lactone; xyloccensins } \mathrm{O}-\mathrm{S} \text { and V; xylogranin B; xylogranatopyridines } \mathrm{A} \text { and } \mathrm{B} \text {; xylogranatumines } \\
\text { A-G; xylogranatin E }\end{array}$ & {$[22,26,36,50,52,53]$} \\
\hline Root bark & N-Methylflindersine & [54] \\
\hline
\end{tabular}

used to treat the troubles caused by dysentery, diarrhea, and abdominal pains [48]. In a recent study, 25 new limonoids have been detected using high resolution electrospray ionization mass spectroscopy (HRESIMS) in $X$. granatum of Hainan mangrove region. The structures of these compounds were also established using singlecrystal X-ray diffraction analyses, nuclear magnetic resonance spectroscopy, and electronic circular dichroism spectra. Limonoids showed typical bridges of $\mathrm{C}_{3}-\mathrm{O}-\mathrm{C}_{8}$ and $\mathrm{C}_{1}-\mathrm{O}-\mathrm{C}_{8}$ in mexicanolides, whereas few compounds, which are derivatives of azadirone, showed $\mathrm{C}_{1}-\mathrm{O}-\mathrm{C}_{29}$ bridges (Zahang et al., 2020).

3.5. Pharmacological Investigation. The pharmacological activities of Xylocarpus granatum J. Koenig are summarized in Table 2 .

3.5.1. Anticancer Activity. Cancer is the abnormal proliferation of the human body's cells. The uncontrolled development of normal cells can initiate cancer. In recent studies, the anticancer properties have been found in some plant extracts and their isolated compounds developed by the researchers. Amongst them is $X$. granatum, a well-known mangrove plant, a rich source of bioactive compounds, such as protolimonoids (apotirucallanes) [57, 58], gedunin [16], xylogranatumines A-G [53], protoxylocarpins F-H [25], and so on, which showed anticancer activity. Besides, protolimonoids exhibited a wide range of bioactivities, including insecticidal [59], and CDC25B (M-phase inducer phosphatase 2) inhibitory properties [60]. The cytotoxic activity of gedunin extracted from bark of $X$. granatum demonstrated moderate levels of anticancer activity in colon cancer cell lines with $\mathrm{IC}_{50}$ value of $16.83 \mu \mathrm{M}$ concentration. In this study, gedunin moderately inhibited $\mathrm{CaCo} 2$ (human colorectal adenocarcinoma) and had growth inhibitory activity [16]. Moreover, xylogranatumines A-G (apotirucallane protolimonoids) had the potential to cytotoxic activities against human A549 (lung adenocarcinoma) tumor cell line. However, at $10 \mu \mathrm{M}$ concentration, only xylogranatumine $\mathrm{F}$ showed weak cytotoxic activity against A549 alveolar tumor cell with inhibition of $54.2 \%$ at the concentration of $10 \mu \mathrm{M}$, while others were inactive $(<50 \%$ inhibition at $10 \mu \mathrm{M})$
[53]. Besides, the isolated protoxylocarpins F-H from seed kernels were assessed for cytotoxicity activity against five human tumor cell lines. However, only 7-deacetylgenudin showed cytotoxic activity against lung cancer cell line, that is, CHAGO $\left(\mathrm{IC}_{50}: 16.0 \mu \mathrm{M}\right)$, and hepatocarcinoma cell line, Hep$\mathrm{G} 2\left(\mathrm{IC}_{50}: 10.26 \mu \mathrm{M}\right)$, while xylogranatin $\mathrm{C}$ showed cytotoxic activity against $\mathrm{CHAGO}$ cells $\left(\mathrm{IC}_{50}: 9.16 \mu \mathrm{M}\right)$ and 7-oxo-7deacetoxygenudin depicted anticancer activity against Hep-G2 cells $\left(\mathrm{IC}_{50}: 16.17 \mu \mathrm{M}\right)$ [25]. In human breast carcinoma cells (KT), xylomexicanin A extracted from seed exhibited antiproliferative at $\mathrm{IC}_{50}$ value of $7.43 \mu \mathrm{M}$ [32], and xylomexicanin $\mathrm{F}$ showed moderate antiproliferative activity against human tumor cell lines A549 and RERF at 18.83 and $15.83 \mu \mathrm{M}$ concentration, respectively [44]. Furthermore, granaxylocarpins $\mathrm{A}$ and $\mathrm{B}$ showed weak cytotoxic effect against the P-388 murine leukemia cell lines at $\mathrm{IC}_{50}$ values of 9.3 and $4.9 \mu \mathrm{M}$, respectively, but inactive against the A549 cell line (IC50 values $>10 \mu \mathrm{M}$ ). In addition, granaxylocarpins $C, D$, and $E$ were inactive against both the P-388 and A-549 cell lines [30]. Besides, antitumor activities of thaixylogranins A-H exhibited weak cytotoxicity against the breast MDA-MB-231 cell line [61], with the concentration of $\mathrm{IC}_{50}$ values of $49.4,58.3,53.6,61.1,57.9$, $44.6,40.6$, and $38.5 \mathrm{mM}$, respectively, whereas thaixylogranin E showed weak cytotoxicity against the HCT-8/ $\mathrm{T}$ cell with an $\mathrm{IC}_{50}$ value of $36.4 \mathrm{mM}$. Compound thaixylogranins $\mathrm{C}$ and $\mathrm{D}$ exhibited weak cytotoxicity against the melanoma A375 cell with $\mathrm{IC}_{50}$ values of 47.1 and $41.9 \mathrm{mM}$, respectively; and in gastric cancer AGS cell line, thaixylogranins $C$ and D showed same cytotoxic effect with IC50 values of 41.7 and $35.0 \mathrm{mM}$, respectively [29]. Xylogranin B was isolated from $X$. granatum leaves, could inhibit T cell factor (TCF)/ $\beta$-catenin transcriptional activity at $\mathrm{IC}_{50}$ $48.9 \mathrm{nM}$, and exhibited strong cytotoxicity against colon cancer cell lines. Xylogranin B drastically attenuated $\beta$-catenin protein; these results indicated that Wnt signal inhibitory effects [26]. All of these compounds were isolated from different parts of $X$. granatum. Xylocarpus granatum leaf extracts (ethyl acetate and its 1-7 fractions) had anticancer activity against HeLa (human cervical adenocarcinoma), T47D (breast cancer), and HT-29 cell (human colorectal adenocarcinoma cell line), with fraction 5 having the most powerful activity of $23.12 \mathrm{ppm} \mathrm{IC}_{50}$ value. However, it was concluded that, to understand the mechanism, further studies are essential [62]. 
<smiles>C=C1C(=O)N(C)c2ccccc2-c2[nH]c(=O)ccc21</smiles>

N-methylflindersine

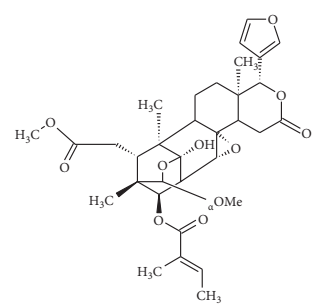

Sundarbanxylogranins C

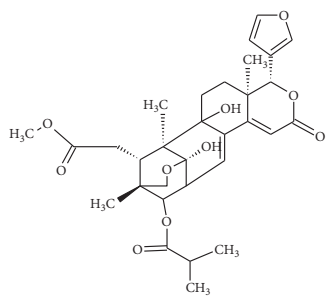

krishnagranagtin B

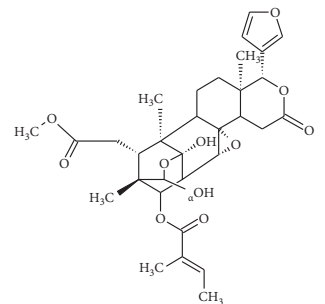

krishnagranagtin $\mathrm{F}$

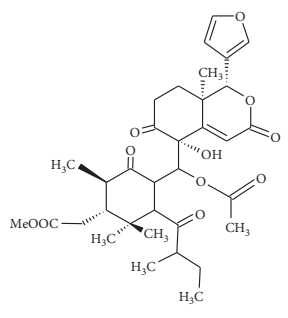

Granaxylocarpin A

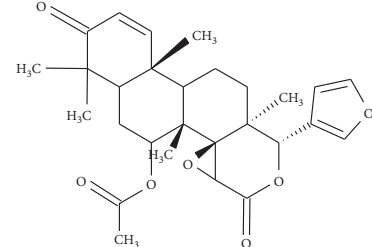

Gedunin

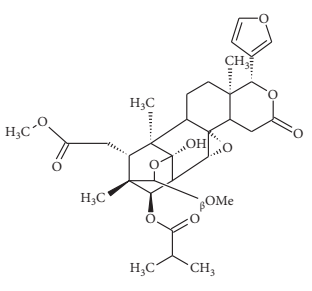

Sundarbanxylogranins D

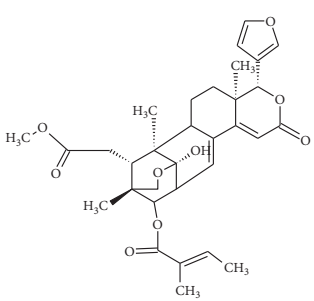

krishnagranagtin C

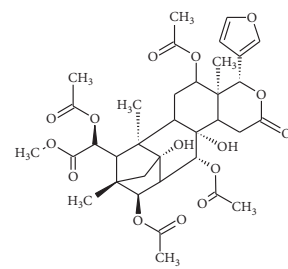

krishnagranagtin $\mathrm{G}$

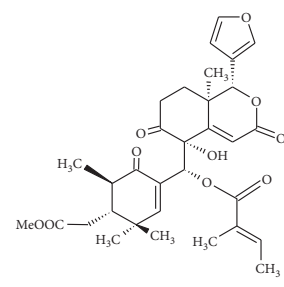

Granaxylocarpin B

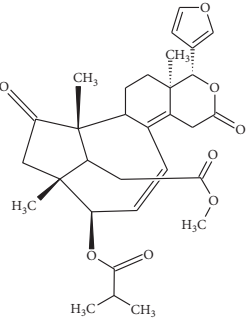

Sundarbanxylogranins A

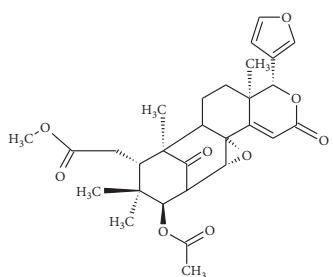

Sundarbanxylogranins B

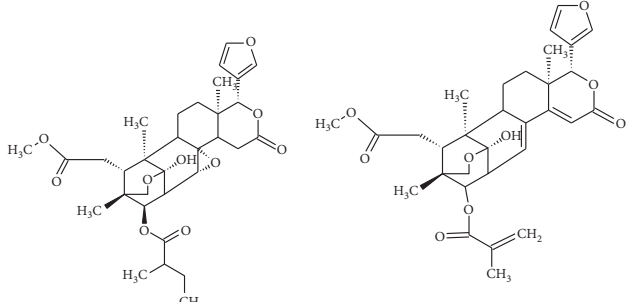

Sundarbanxylogranins E

krishnagranagtin A

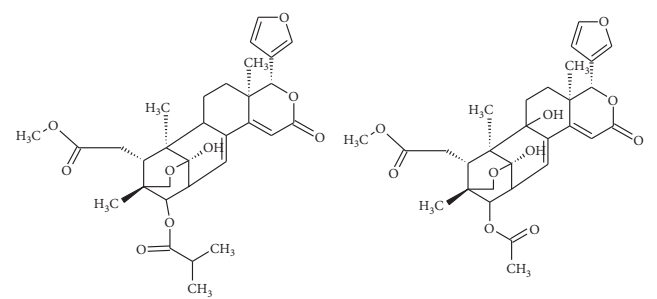

krishnagranagtin D

krishnagranagtin E
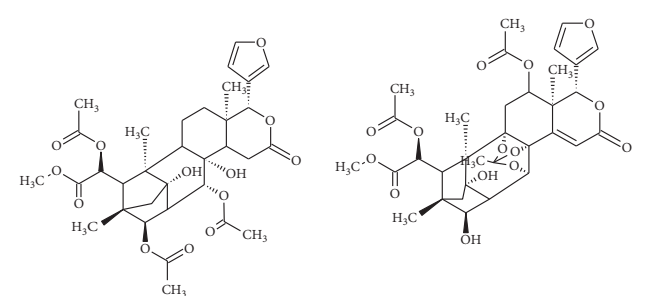

krishnagranagtin $\mathrm{H}$

krishnagranagtin I

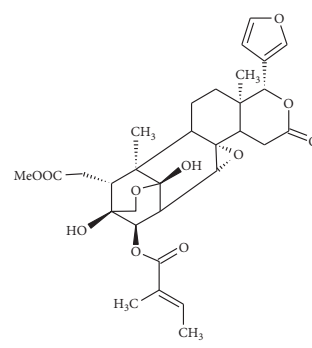

Granaxylocarpin C

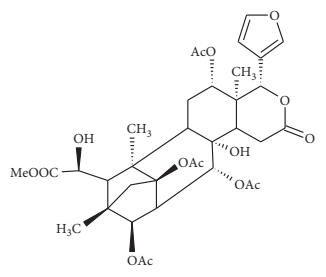

Granaxylocarpin D

(a)

Figure 2: Continued. 


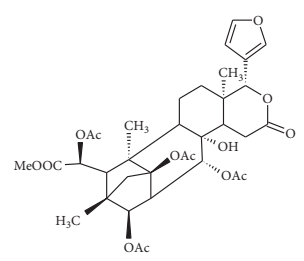

Granaxylocarpin E

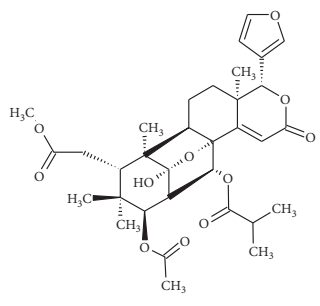

Xylomexicanin D

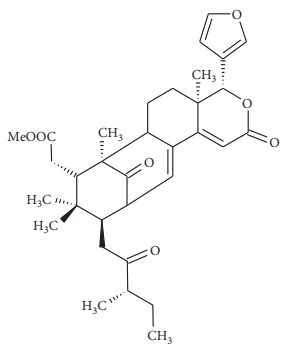

Granatumin B

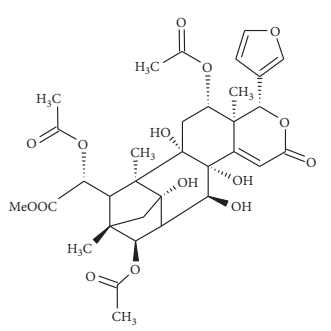

Granatumin F

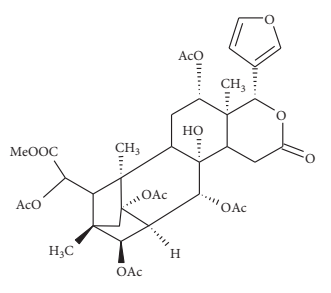

Granatumin J

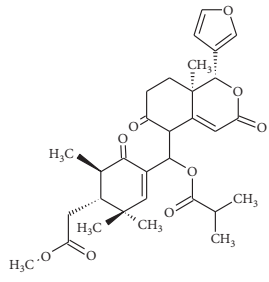

Xylomexicanin A

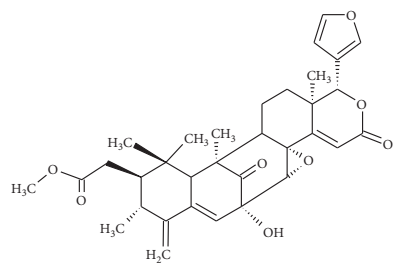

Xylomexicanin I

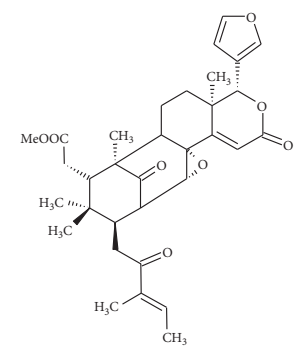

Granatumin C

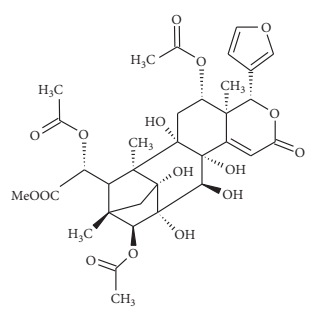

Granatumin G

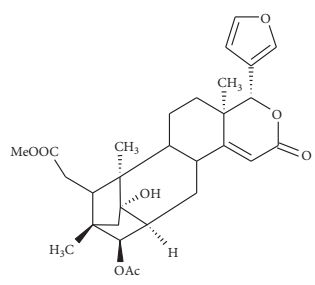

Granatumin K

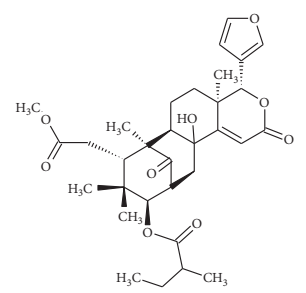

Xylomexicanin B

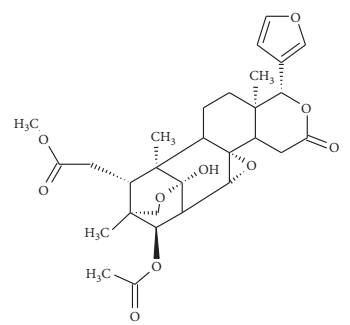

Xylomexicanin J

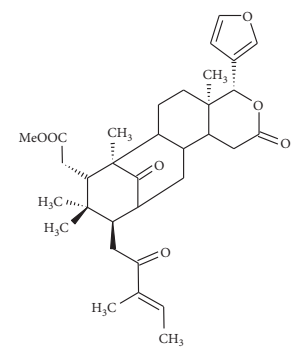

Granatumin D

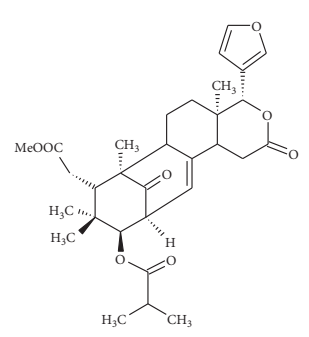

Granatumin H

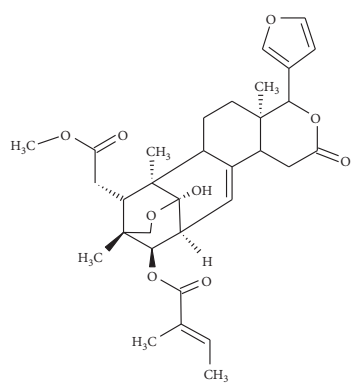

Granatumin L

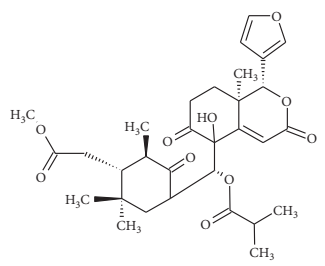

Xylomexicanin C

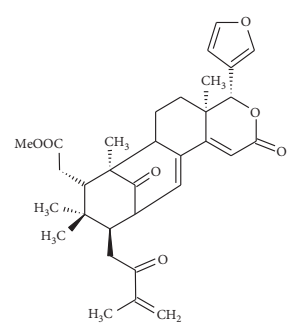

Granatumin A

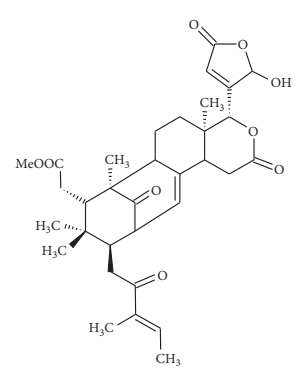

Granatumin E

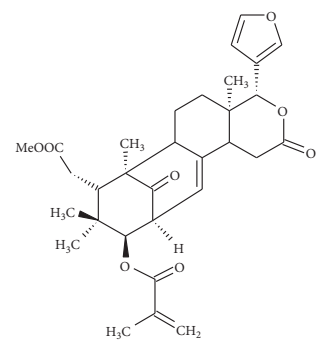

Granatumin I

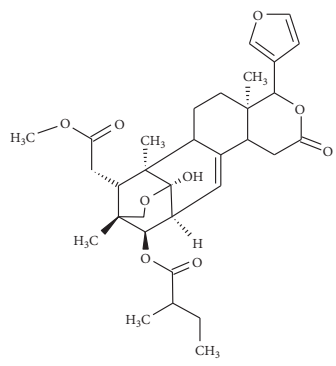

Granatumin M

(b)

Figure 2: Continued. 


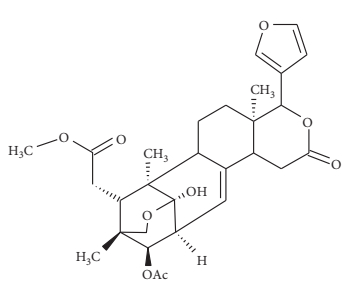

Granatumin N

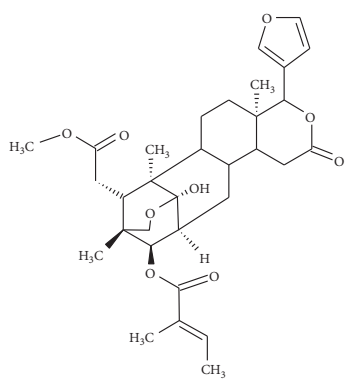

Granatumin R

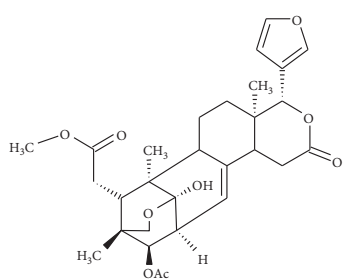

Granatumin V

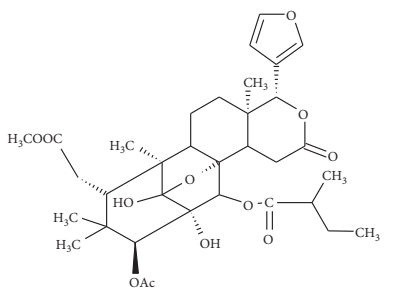

xylocecnsin I

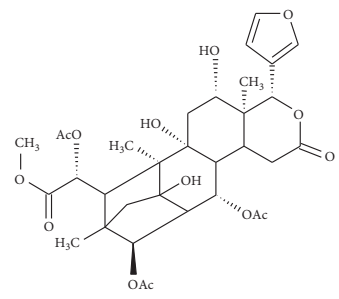

xylocecnsin Y

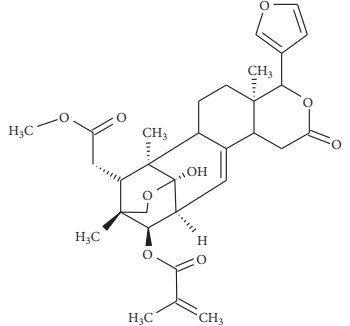

Granatumin O

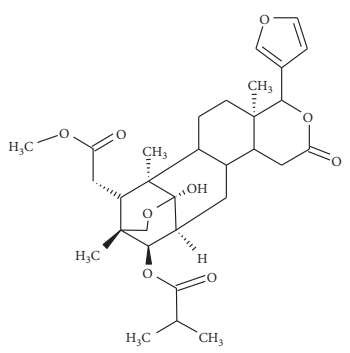

Granatumin S

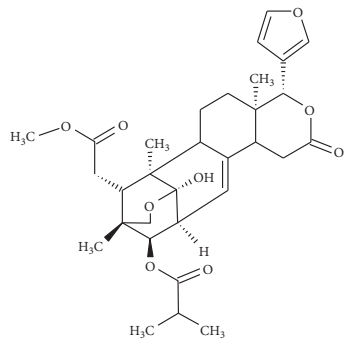

Granatumin W

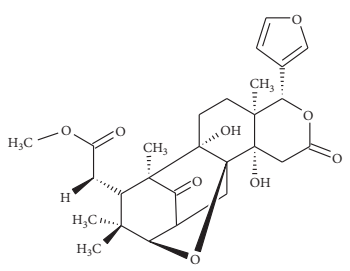

xylocecnsin K
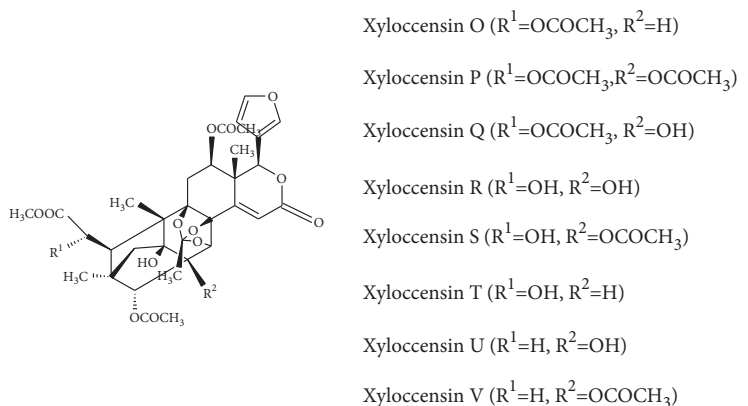

(c)

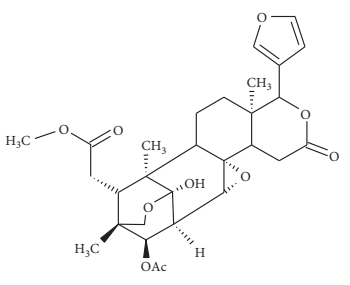

Granatumin P

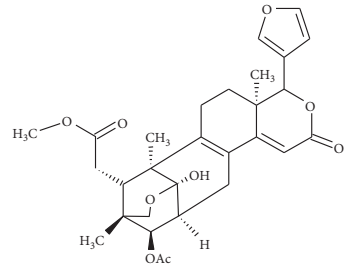

Granatumin T

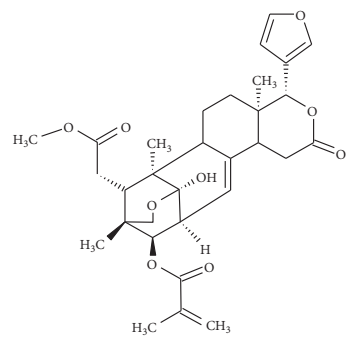

Granatumin X

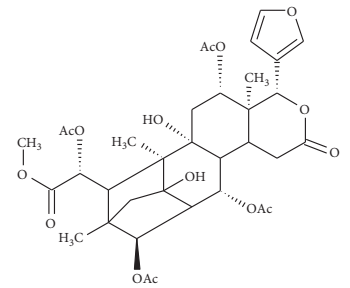

xylocecnsin L

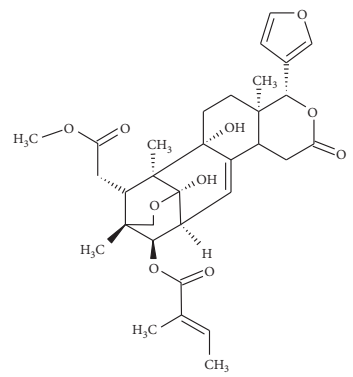

Granatumin Y

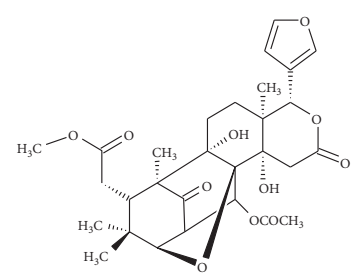

xylocecnsin W

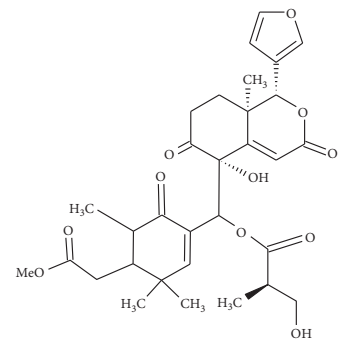

Hainangranatumin A

FIgURE 2: Continued. 


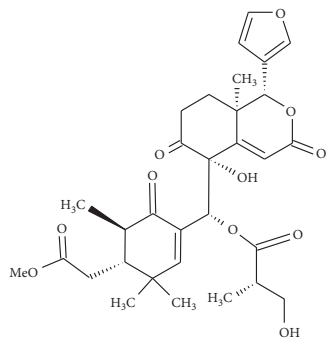

Hainangranatumin B

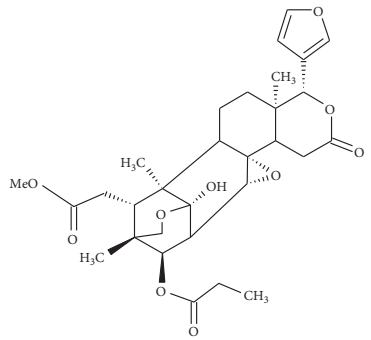

Hainangranatumin $\mathrm{F}$

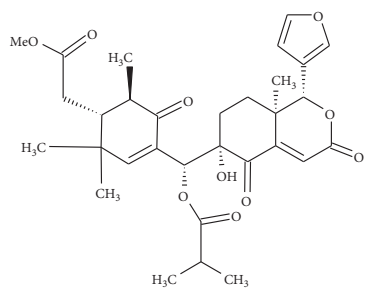

Hainangranatumin J

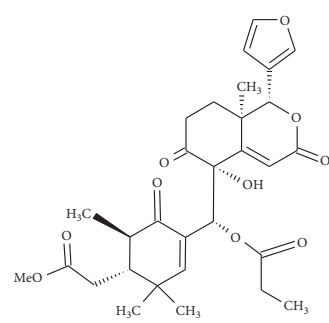

Hainangranatumin C

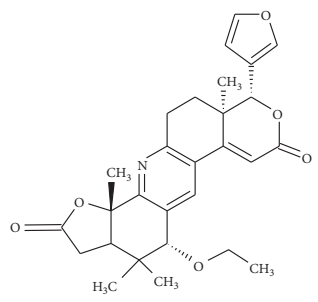

Hainangranatumin G

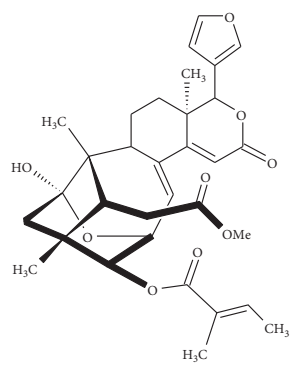

Andhraxylocarpin A

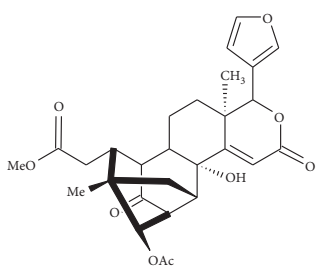

Andhraxylocarpin E

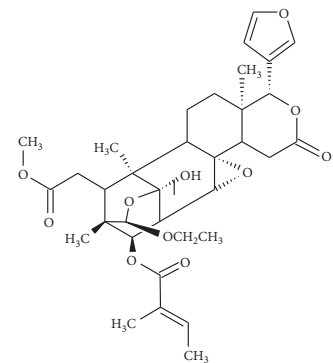

Thaixylogranin D<smiles>COC(=O)C[C@H]1[C@@H](C)C(=O)C([C@H](O)C2C(=O)CC[C@@]3(C)C2CC(=O)O[C@H]3c2ccoc2)=CC1(C)C</smiles>

Hainangranatumin D

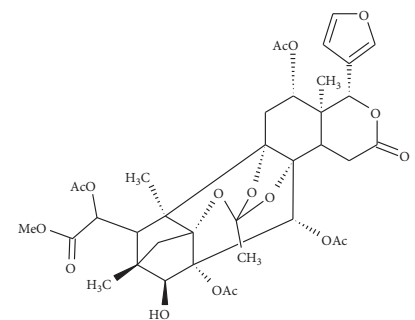

Hainangranatumin $\mathrm{H}$

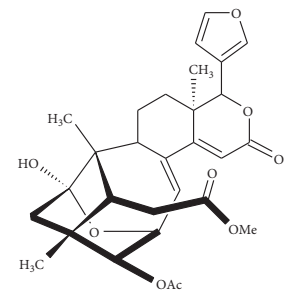

Andhraxylocarpin B

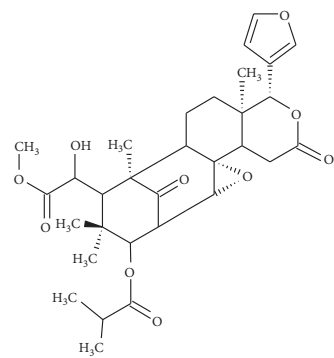

Thaixylogranin A

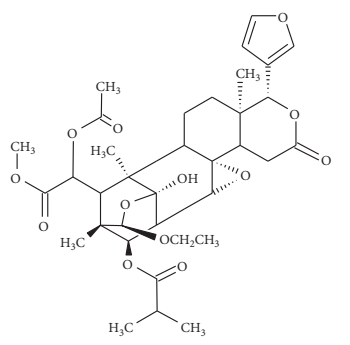

Thaixylogranin E

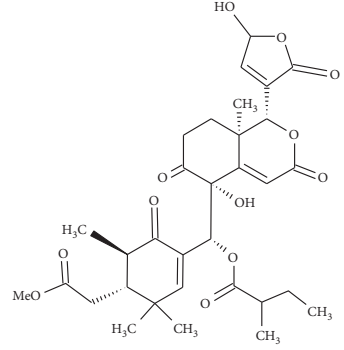

Hainangranatumin E

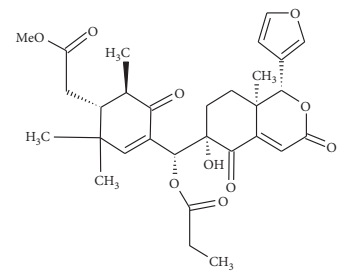

Hainangranatumin I

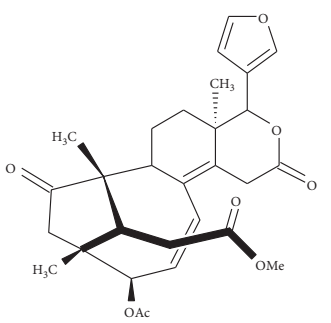

Andhraxylocarpin C

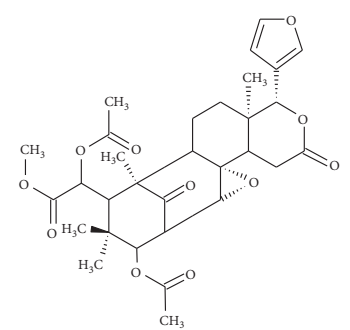

Thaixylogranin B

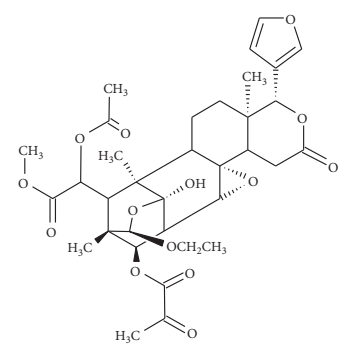

Thaixylogranin F

(d)

Figure 2: Continued. 


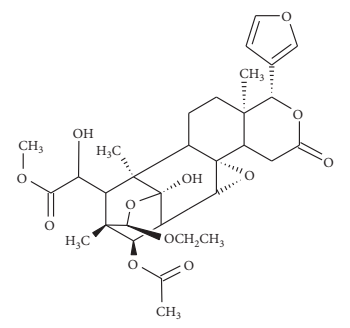

Thaixylogranin G

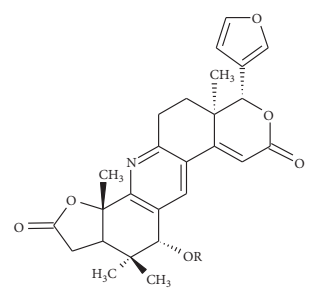

Xylogranatin $\mathrm{F}(\mathrm{R}=\mathrm{H})$ Xylogranatin $\mathrm{G}\left(\mathrm{R}=\mathrm{COCH}_{3}\right)$

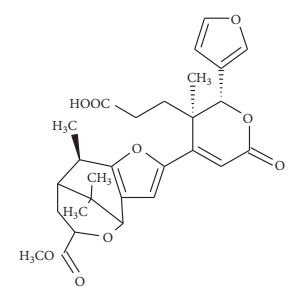

Xylogranatin Q

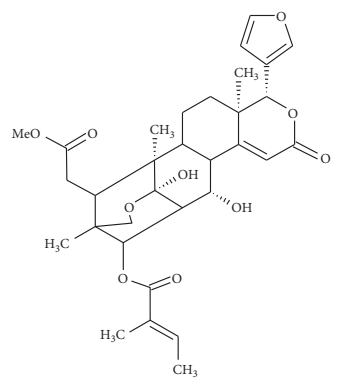

Thaigranatin C

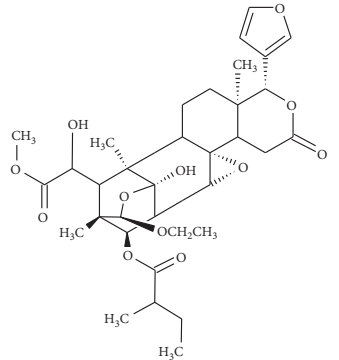

Thaixylogranin $\mathrm{H}$

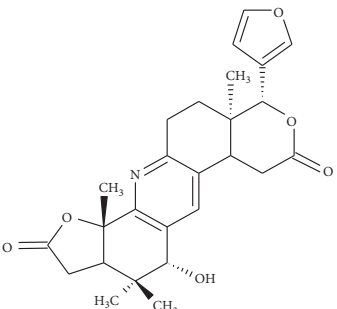

Xylogranatin $\mathrm{H}$<smiles>CCC[C@H]1COC(=O)C=C1C(=O)CC1C[C@H](C)[C@H](CC(=O)O)C(C)C1=O</smiles>

Xylogranatin R

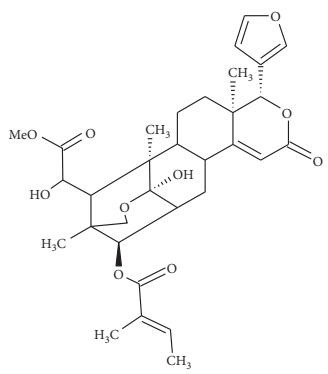

Thaigranatin D

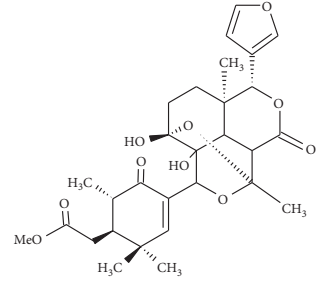

Xylocarponoid A

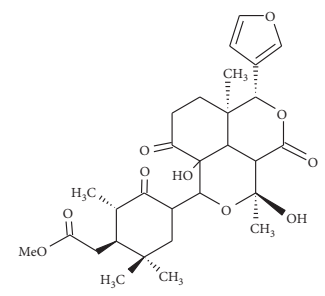

Xylocarponoid B

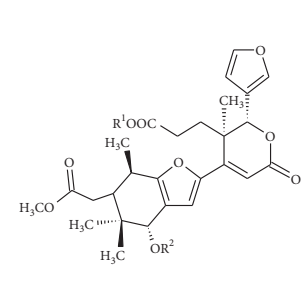

Xylogranatin $\mathrm{I}\left(\mathrm{R}^{1}=\mathrm{H}, \mathrm{R}^{2}=\mathrm{H}\right)$

Xylogranatin $\mathrm{J}\left(\mathrm{R}^{1}=\mathrm{CH}_{3}, \mathrm{R}^{2}=\mathrm{CH}_{3}\right)$

Xylogranatin $\mathrm{K}\left(\mathrm{R}^{1}=\mathrm{H}, \mathrm{R}^{2}=\mathrm{CH}_{3}\right)$

Xylogranatin $\mathrm{L}\left(\mathrm{R}^{1}=\mathrm{H}, \mathrm{R}^{2}=\mathrm{CH}_{2} \mathrm{CH}_{3}\right)$

Xylogranatin $\mathrm{M}\left(\mathrm{R}^{1}=\mathrm{CH}_{3}, \mathrm{R}^{2}=\mathrm{COCH}_{3}\right)$

Xylogranatin $\mathrm{N}\left(\mathrm{R}^{1}=\mathrm{H}, \mathrm{R}^{2}=2 \mathrm{~S}\right.$-methylbutyryl)

Xylogranatin $\mathrm{O}\left(\mathrm{R}^{1}=\mathrm{H}, \mathrm{R}^{2}=\right.$ tigloyl $)$

Xylogranatin $P\left(R^{1}=H, R^{2}=\right.$ isobutyryl $)$

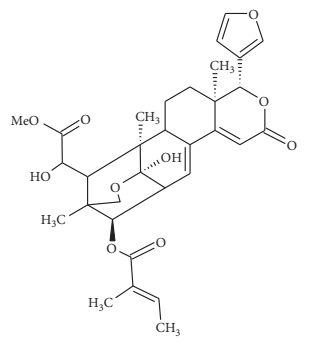

Thaigranatin A

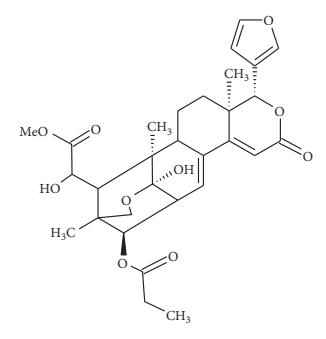

Thaigranatin B

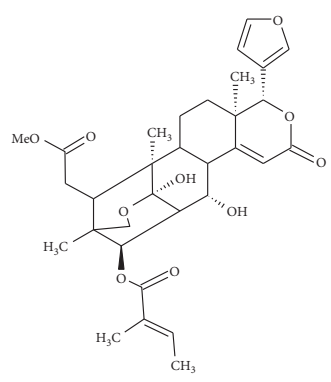

Thaigranatin E

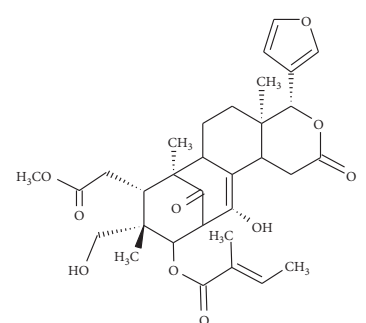

Xylocartin C

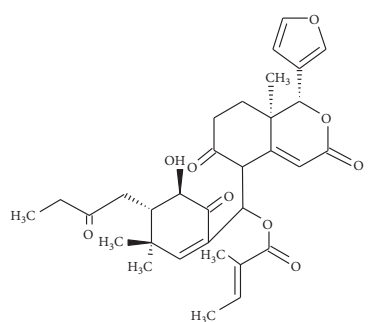

Xylocarpin $\mathrm{H}$

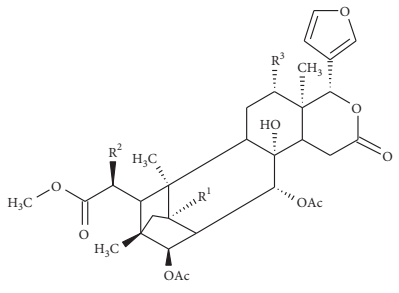

Xylocarpin A (R1=R2=OAc, R3=H)

Xylocarpin B (R1=OAc, R2=R3=H)

Xylocarpin $\mathrm{C}(\mathrm{R} 1=\mathrm{OH}, \mathrm{R} 2=\mathrm{H}, \mathrm{R} 3=\mathrm{OAc})$

Xylocarpin $\mathrm{D}(\mathrm{R} 1=\mathrm{R} 3=\mathrm{OAc}, \mathrm{R} 2=\mathrm{OH})$

Xylocarpin $\mathrm{E}(\mathrm{R} 1=\mathrm{R} 2=\mathrm{OAc}, \mathrm{R} 3=\mathrm{OH})$

(e)

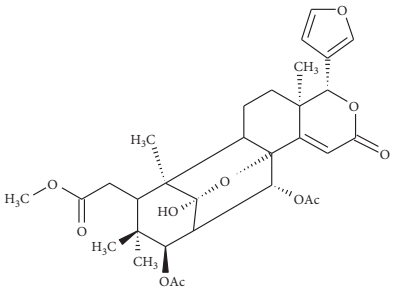

Xylocarpin F

Figure 2: Continued. 

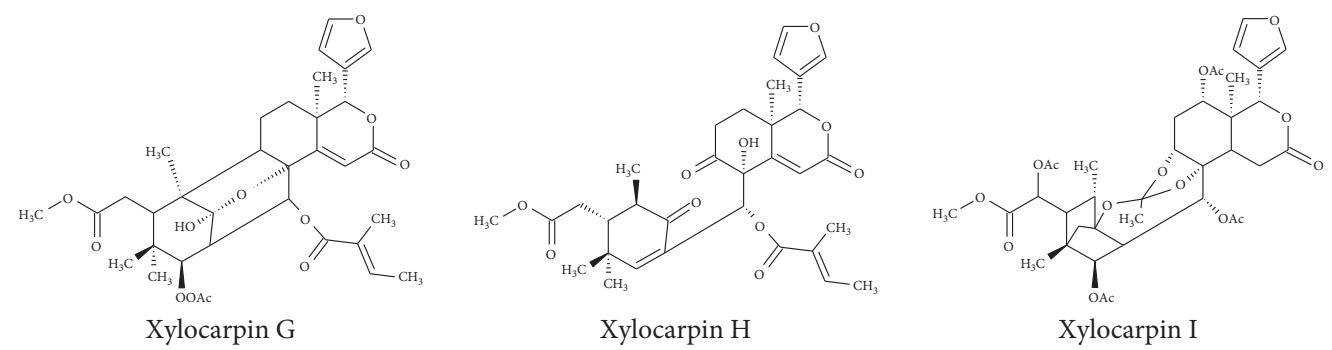

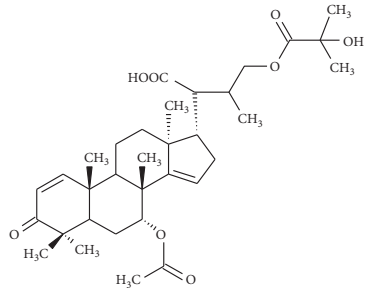

Protoxylogranatin B

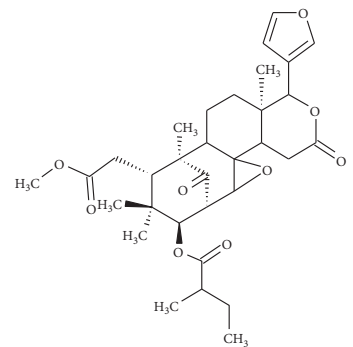

Cipadesin A<smiles>Cc1nc(N)c2c(C)c(I)c(I)c(=O)n2c1I</smiles>

Xylogranatinin

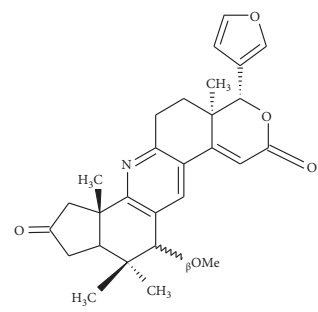

Xylogranatopyridine A

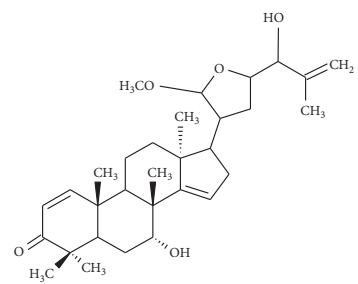

Protoxylogranatin A

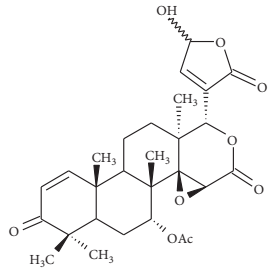

Photogedunin

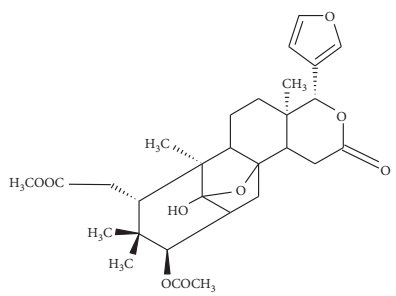

Xyloccensin M

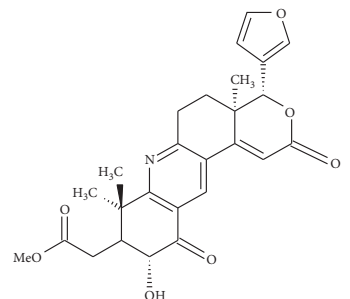

Xylogranatopyridine B

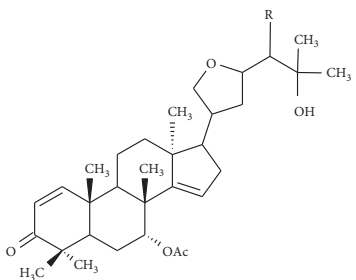

Protoxylocarpin $\mathrm{F}(\mathrm{R}=\beta \mathrm{OH})$

Protoxylocarpin $\mathrm{G}(\mathrm{R}=\mathrm{aOH})$

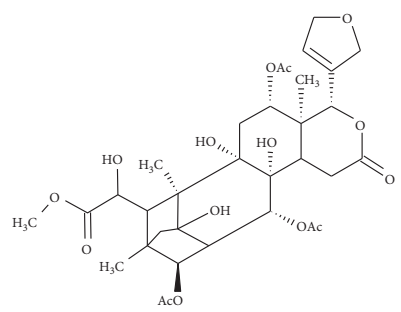

Xylocarpin L

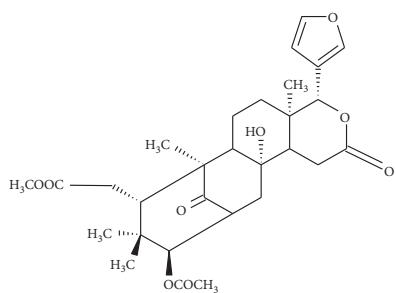

Xyloccensin N

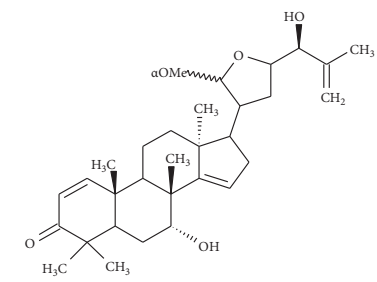

Xylogranatumine A

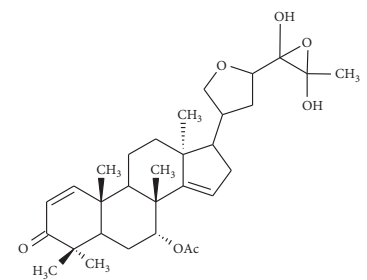

Protoxylogranatin $\mathrm{H}$

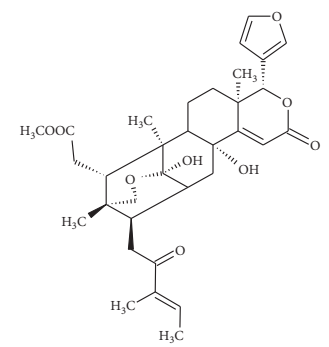

Xylogranatin E

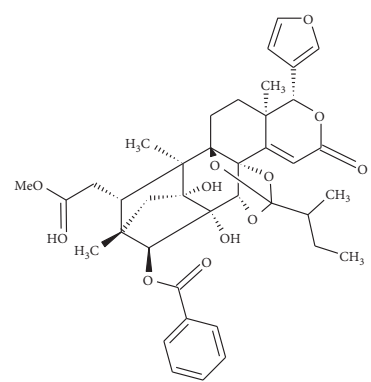

Xylogranin B

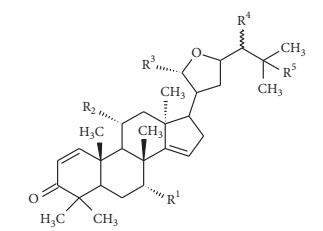

Xylogranatumine $B\left(\mathrm{R}^{1}=\mathrm{OAc}, \mathrm{R}^{2}=\mathrm{H}\right.$, $\mathrm{R}^{3}=\mathrm{OMe}, \mathrm{R}^{4}=\beta \mathrm{OH}, \mathrm{R}^{5}=\mathrm{OH}$ )

Xylogranatumine $\mathrm{C}\left(\mathrm{R}^{1}=\mathrm{OH}, \mathrm{R}^{2}=\mathrm{H}\right.$, $\mathrm{R}^{3}=\mathrm{OMe}, \mathrm{R}^{4}=\alpha \mathrm{OAc}, \mathrm{R}^{5}=\mathrm{OH}$ )

Xylogranatumine $\mathrm{E}\left(\mathrm{R}^{1}=\mathrm{OH}, \mathrm{R}^{2}=\mathrm{OAc}\right.$, $\mathrm{R}^{3}=\mathrm{OMe}, \mathrm{R}^{4}=\alpha \mathrm{OH}, \mathrm{R}^{5}=\mathrm{OMe}$ )

(f)

Figure 2: Continued. 


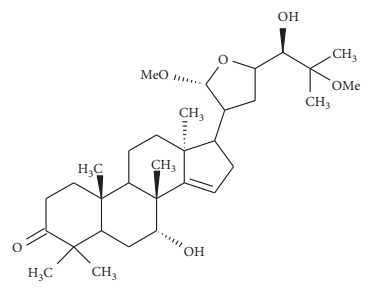

Xylogranatumine D

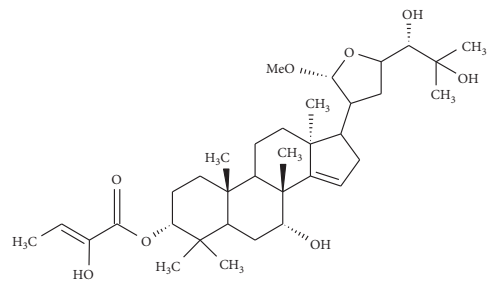

Xylogranatumine $\mathrm{F}$

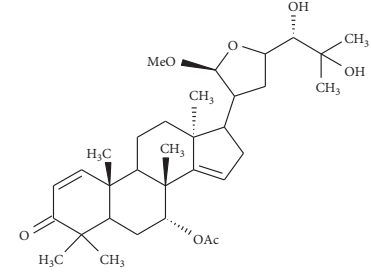

Xylogranatumine $\mathrm{G}$

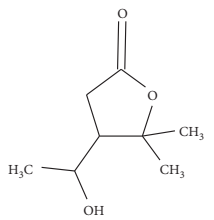

Lactone

(g)

Figure 2: Chemical constituents of Xylocarpus granatum J. Koenig.

TABle 2: Pharmacological activities.

\begin{tabular}{|c|c|c|c|c|}
\hline Activity & Isolated compounds & Cell lines/test system & $\mathrm{IC}_{50}$ values & References \\
\hline \multirow{8}{*}{ Anticancer activity } & Gedunin & $\begin{array}{l}\text { Colon cancer cell lines } \\
\text { A549 (human lung }\end{array}$ & $16.83 \mu \mathrm{M}$ & {$[16]$} \\
\hline & Xylogranatumines $\mathrm{A}-\mathrm{G}$ & $\begin{array}{c}\text { adenocarcinoma) tumor cell } \\
\text { lines }\end{array}$ & $10 \mu \mathrm{M}$ per specimen & {$[53]$} \\
\hline & Protoxylocarpins F-H & Five human tumor cell lines & & {$[25]$} \\
\hline & Xylomexicanin A & $\begin{array}{c}\text { Human breast carcinoma cells } \\
\qquad(\mathrm{KT})\end{array}$ & $7.43 \mu \mathrm{M}$ & {$[32]$} \\
\hline & Granaxylocarpins A-E & $\begin{array}{l}\text { Cytotoxicity against the P-388 } \\
\text { and A-549 tumor cell lines }\end{array}$ & 9.3 and $4.9 \mu \mathrm{M}$ & {$[30]$} \\
\hline & Xylomexicanins F & $\begin{array}{c}\text { Six human tumor cell lines } \\
\text { A549 and RERF }\end{array}$ & 18.83 and $15.83 \mu \mathrm{M}$ & {$[44]$} \\
\hline & Thaixylogranis A-H & MDA-MB-231 cell line & $49.4 \mathrm{mM}$ & [29] \\
\hline & Xylogranin B & & $48.9 \mathrm{nM}$ & {$[26]$} \\
\hline Antimalarial activity & Gedunin and xyloccensin I & $\begin{array}{l}\text { In vitro model of Plasmodium } \\
\text { falciparum }\end{array}$ & $50 \mu \mathrm{g} \mathrm{m} / \mathrm{L}$, and (MIC) $10 \mu \mathrm{g} \mathrm{m} / \mathrm{L}$. & {$[12]$} \\
\hline \multirow{2}{*}{ Antiviral activity } & Sundarbanxylogranis B & $\begin{array}{c}\text { Human immunodeficiency } \\
\text { virus (HIV) }\end{array}$ & $78.45 \pm 1.69 \mu \mathrm{M}$ & {$[27]$} \\
\hline & $\begin{array}{l}\text { Granatumin } \mathrm{L} \text { and their } \\
\text { moderate derivatives }\end{array}$ & $\begin{array}{l}\text { HIV-1 and influenza A virus } \\
\text { (IAV) }\end{array}$ & $\begin{array}{l}\text { HIV-1 with an } 15.98 \pm 6.87 \mu \mathrm{M} \text {; IAV } \\
\text { with a } 14.02 \pm 3.54 \mu \mathrm{M}\end{array}$ & {$[39]$} \\
\hline \multirow[t]{2}{*}{ Antifeedant activity } & Xylogranatins G & $\begin{array}{l}\text { The third instar larvae of } \\
\text { Mythimna separata (walker) }\end{array}$ & $1 \mathrm{mg} \mathrm{m} / \mathrm{L}$ & {$[38]$} \\
\hline & Xyloccensins P and Q & & $500 \mathrm{ppm}$ & {$[10]$} \\
\hline \multirow{3}{*}{ Antidiabetic activity } & Xylogranin B & & $\begin{array}{c}\mathrm{IC}_{50} 48.9 \mathrm{nM} \text { or inhibited TCF/ } \\
\beta \text {-catenin transcriptional activity } \\
\text { with IC50 values of } 270 \text { and } 330 \mathrm{nM}\end{array}$ & {$[26]$} \\
\hline & Xyloccensin I & $\begin{array}{l}\alpha \text {-amylase and } \alpha \text {-glucosidase } \\
\text { inhibition study }\end{array}$ & 0.25 and $0.16 \mathrm{mg} / \mathrm{mL}$ & {$[4]$} \\
\hline & Xyloccensin S & $\begin{array}{l}\text { Protein tyrosine phosphatase } \\
\text { 1B }\end{array}$ & $8.72 \mu \mathrm{g} / \mathrm{mL}$ & {$[52]$} \\
\hline $\begin{array}{l}\text { Antidepressant- and } \\
\text { anxiolytic-like activities }\end{array}$ & Cipadesin A & Mice model & 5,15 , and $50 \mathrm{mg} / \mathrm{kg}$ & {$[2]$} \\
\hline Antifilarial activity & Gedunin and photogedunn & $\begin{array}{l}\text { Human lymphatic filarial } \\
\text { parasite }\end{array}$ & $100 \mathrm{mg} / \mathrm{kg}$ & {$[56]$} \\
\hline Fungicidal activity & Lactone & Wheat powdery mildew & $20 \mathrm{mg} / \mathrm{mL}$ & {$[22]$} \\
\hline \multirow{3}{*}{$\begin{array}{l}\text { Possess different } \\
\text { inhibitory effect }\end{array}$} & $\begin{array}{c}\text { Xylogranatopyridins A and } \\
\text { B }\end{array}$ & $\begin{array}{c}\text { Protein tyrosinephosphatase } \\
\text { 1B (PTP1B) }\end{array}$ & $22.9 \mu \mathrm{M}$ & {$[52]$} \\
\hline & $\begin{array}{c}\text { Krishnagranatins } G, \mathrm{H} \text {, and } \\
\text { I }\end{array}$ & Inhibit NF- $\kappa$ B pathway & $100 \mathrm{ng} / \mathrm{mL}$ & {$[28]$} \\
\hline & Xyloccensin S & $\begin{array}{l}\text { Protein tyrosine phosphatase } \\
\text { 1B }\end{array}$ & $8.72 \mu \mathrm{g} / \mathrm{mL}$ & {$[36]$} \\
\hline Antioxidant activity & Xyloccensin I & $\begin{array}{l}\text { DPPH, ABTS, superoxide and } \\
\text { hydrogen peroxide scavenging }\end{array}$ & $0.041,0.039,0.096$, and $0.235 \mathrm{mg} / \mathrm{mL}$ & {$[4]$} \\
\hline
\end{tabular}


3.5.2. Antimalarial Activity. Mosquitoes are the key vectors for ruining parasites and pathogens including malaria, dengue, filariasis, yellow fever, chikungunya, encephalitis, and so on [63]. Malaria is a global health problem, and about 300-500 million people are infected, while almost 1 million people die annually. Natural products may be a choice for the treatment of malaria [64]. The isolated compounds from $X$. granatum plant had potential antimalarial activity [65]. The compounds gedunin and xyloccensin I, isolated from $X$. granatum, had antimalarial activity at the concentration of $50 \mu \mathrm{gm} / \mathrm{L}$ only and showed a minimum inhibitory concentration (MIC) of $10 \mu \mathrm{g} \mathrm{m} / \mathrm{L}$. This antimalarial activity was exhibited by killing the parasites in in vitro model of Plasmodium falciparum [12].

3.5.3. Antiviral Activity. A virus is a particle that replicates within the living cells of an organism. Viruses can infect all types of life forms, including animals, plants, and microorganisms (like bacteria and archaea). The medicinal plant should be a good source of antiviral agents. X. granatum plant extract contains a variety of bioactive compounds that act against virus including sundarbanxylogranins $\mathrm{B}$ and granatumin L [27, 39]. Hence, sundarbanxylogranins B showed moderate effect against human immunodeficiency virus (HIV) with the concentration of $20 \mu \mathrm{M}$; it showed a moderate inhibitory rate of $58.14 \pm 3.67 \%$ and the values of $\mathrm{IC}_{50}$ and $\mathrm{CC}_{50}$ for sundarbanxylogranin B were 23.14 \pm 1.29 and $78.45 \pm 1.69 \mu \mathrm{M}$, respectively [27]. Besides, in vitro antiviral activities of granatumin $\mathrm{L}$ and its derivatives against HIV-1 and influenza A virus (IAV) were evaluated. Granatumin $\mathrm{L}$ and their derivatives exhibit activities against the HIV-1 with an $\mathrm{IC}_{50}$ value of $15.98 \pm 6.87 \mu \mathrm{M}$ and a $\mathrm{CC}_{50}$ value greater than $100.0 \mu \mathrm{M}$, whereas its derivative showed significant inhibitory activity against $\mathrm{IAV}$ with an $\mathrm{IC}_{50}$ value of $14.02 \pm 3.54 \mu \mathrm{M}$ and $\mathrm{CC}_{50}$ value greater than $100.0 \mu \mathrm{M}$. Thus, it was inferred by the authors that alkyl groups at the C-3 position helped in exhibiting the antiviral activity against HIV [39].

3.5.4. Antifeedant Activity. Some plants are used to treat several diseases and disorders. Antifeedant agents are natural substance, which stops or inhibits feeding by a pest, especially an insect [66]. Natural compounds should be the trusted source to control pests. $X$. granatum plant contains a number of isolated compounds, like secondary metabolites, and has the defense capability [67]. Several phytoconstituents and xylogranatins F, G, and R are isolated from seeds of the Chinese mangrove, $X$. granatum plant. These compounds exhibit good antifeedant activity against third instar larvae of Mythimna separata (Walker) at $1 \mathrm{mg} / \mathrm{mL}$ concentration [38]. Among of them, xylogranatin $G$ was the most potent compound at 0.31 and $0.30 \mathrm{mg} / \mathrm{mL}$ concentration and 24 and $48 \mathrm{~h}$; it showed $50 \%$ antifeedant activity median antifeedant concentration (AFC50), respectively [38]. Furthermore, both xyloccensins P and Q, isolated from $X$. granatum plant, showed potent antifeedant activity at $500 \mathrm{ppm}$ concentration against third instar larvae of
M. separata, whereas other compounds xyloccensins $\mathrm{O}$ and $\mathrm{R}-\mathrm{V}$ showed weak activity [10].

3.5.5. Antidiabetic Activity. Diabetes is a condition, in which the body's blood glucose is higher than the normal level [68]. Blood glucose is controlled by a vital hormone called insulin and depending on insulin the diabetes is divided into two major categories: types 1 and 2 diabetes $[68,69]$. The isolated compounds from the $X$. granatum have antidiabetic activity. Xylogranin B, xyloccensin I, and xyloccensin S [70] exhibited antidiabetic activity by inhibiting $\alpha$-amylase, $\alpha$-glucosidase, and protein tyrosine phosphatase $1 \mathrm{~B}$ (PTP1B), respectively $[4,52]$. Xylogranin $\mathrm{B}$ acts with the concentration of the $\mathrm{IC}_{50} 48.9 \mathrm{nM}$ or inhibited $\mathrm{TCF} / \beta$-catenin transcriptional activity with $\mathrm{IC}_{50}$ values of 270 and $330 \mathrm{nM}[4,26]$. Besides this other compound, xyloccensin S can control blood glucose levels with the $\mathrm{IC}_{50}$ value of $8.72 \mu \mathrm{g} / \mathrm{mL}$ in PTP1B in vitro study [52]. In addition, xyloccensin I exhibited a hypoglycemic effect on $\alpha$-amylase and $\alpha$-glucosidase inhibition study at the concentrations of 0.25 and $0.16 \mathrm{mg} / \mathrm{mL}$, respectively [4]. Hence, compounds present in the $X$. granatum have inhibitory effect on the enzymes ( $\alpha$-amylase and $\alpha$-glucosidase) that increase the level of blood glucose in body. Inhibition of $\alpha$-amylase and $\alpha$-glucosidase plant extracts lead to reduction in the level of blood sugar and helps in management of diabetic condition [71-73].

\subsubsection{Antidepressant- and Anxiolytic-Like Activities.} Anxiety and depression are generally a normal reaction to stress and there will always be situations that create stress and discomfort in humans for several reasons [74]. These disorders are currently considered the most common psychiatric illnesses affecting humans [75]. It is one of the most common psychiatric disorders. Information collected from the WHO states that depression is expected to become the second leading cause of disease-related disability by the year 2020, following heart disease [76]. Current pharmacological interventions suggest that the drugs used to manage these disorders, often have a number of side effects, including drug interactions, delayed response, and even nonresponse to the treatment [77]. Natural compounds have potential antidepressant- and anxiolytic-like activities. Recent studies exhibited that cipadesin A extracted from the fruits of the $X$. granatum plant assert potential antidepressant- and anxiolytic-like activities [2]. In the forced swimming test (FST), cipadesin A treatment significantly decreased the floating time in in vitro testing model. Cipadesin A treatment (15 and $50 \mathrm{mg} / \mathrm{kg}$ doses) drastically reduced the floating time [2]. Moreover, in the tail suspension test, administering 15 and $50 \mathrm{mg} / \mathrm{kg}$ doses cipadesin A significantly decreased the immobility time. Furthermore, at 5, 15, and $50 \mathrm{mg} / \mathrm{kg}$ doses significantly increased the time spent in the central zone of mice. Additionally, cipadesin A at 15 and $50 \mathrm{mg} / \mathrm{kg}$ doses significantly decreased serum corticosterone and adrenocorticotropic hormone levels [2]. The study found that after seven days, cipadesin A administered orally 
exhibited significant antidepressant-like effect in the tail suspension and forced swimming tests in mice [2].

3.5.7. Antifilarial Activity. Filariasis is a parasitic disease caused by an infection with roundworms of the Filarioidea type [78]. These are spread by blood-feeding insects such as black flies and mosquitoes [79]. They belong to the group of diseases called helminthiases. Plants may be an effective source of antifilarial agents. Research findings proposed that photogedunin isolated from the fruits of the $X$. granatum plant administered by a subcutaneous route at IC50 of 0.239 and $0.213 \mu \mathrm{g} / \mathrm{mL}$ and CC50 of 212.5 and $262.3 \mu \mathrm{g} / \mathrm{mL}$, respectively, at $5 \times 100 \mathrm{mg} / \mathrm{kg}$ revealed excellent antifilarial efficacy, resulting in the death of $80 \%$ and $70 \%$ transplanted adult Brugia malayi in the peritoneal cavity of jirds, respectively [56].

3.5.8. Antifungal Activity. Fungus infection is a major global health problem, and these life-threatening causative agents create approximately 1.5 billion deaths annually [80]. To prevent this infection, we found out some phytochemicals from natural plants. Lactone isolated from leaves of $X$. granatum plant showed $67.4 \%$ inhibition rate, and a strong fungicidal activity was proved against wheat powdery mildew with the $20 \mathrm{mg} / \mathrm{mL}$ concentration [22]. The petroleum ether and ethyl acetate extracts of $X$. granatum showed the presence of tricontanol, kaempferol, and sitosterol, which can easily penetrate the cellular barrier of powdery mildew fungus and creates pore, ultimately leading to the leakage of electrolyte causing cell death.

3.5.9. Different Inhibitory Effects. The inhibitory activity was shown against PTP1B by xylogranatopyridines A and B isolated from the twigs and leaves of $X$. granatum plant. The inhibitory concentration of the chemical was an $\mathrm{IC}_{50}$ value of $22.9 \mu \mathrm{M}$ [52]. At the concentration of $10.0 \mu \mathrm{M}$, krishnagranatins $\mathrm{G}, \mathrm{H}$, and I drastically inhibited the activation of nuclear factor- (NF-) $\kappa \mathrm{B}$, induced by lipopolysaccharides (LPS) at the concentration of $100 \mathrm{ng} / \mathrm{mL}$ [28]. However, these compounds implied that the previously mentione NF$\kappa \mathrm{B}$ inhibitory activity was not related to cell death. In conclusion, krishnagranatins $\mathrm{G}, \mathrm{H}$, and I displayed a significant inhibitory effect against the activation of $N F-\kappa B$ signaling pathways [28]. Furthermore, xyloccensin $\mathrm{S}$ followed inhibitory activity against PTP1B at the amount of $\mathrm{IC}_{50}$ value of $8.72 \mu \mathrm{g} / \mathrm{mL}$ [36].

3.5.10. Antioxidant Activity. The antioxidant constituents from ethanol bark extract of a medicinally important mangrove plant $X$. granatum [4]. Limonoid derivative xyloccensin I showed antioxidant activity in 2,2-diphenyl-1picrylhydrazyl (DPPH), 2,2'-azino-bis(3-ethylbenzothiazoline-6-sulfonic acid) (ABTS), superoxide, and hydrogen peroxide scavenging studies at IC50 values of $0.041,0.039$, 0.096 and $0.235 \mathrm{mg} / \mathrm{mL}$, respectively [4]. In a recent study by Das and coworkers [81], demonstrated that bark ethanolic extract reflected highest ABTS scavenging activity with $\mathrm{IC}_{50}$ value of $41.50 ? \mathrm{~g} / \mathrm{mL}$, whereas butylated hydroxyl toluene (standard antioxidant) demonstrated antioxidant activity of 76.34 ?g/mL. In vivo antioxidant analysis showed that enzymatic antioxidants such as superoxide dismutase, catalase, and glutathione reductase in liver and brain tissue of diabetic mice increased when supplemented with $200 \mathrm{mg} / \mathrm{kg}$ of $X$. granatum. Hence, it was concluded that increase in the antioxidant defense system helped the diabetic mice in overcoming the oxidative stress.

3.5.11. Antibacterial Effect. Stem bark extracts (ethanol extract, pet-ether fraction, $\mathrm{CCl} 4$ fraction, and $\mathrm{CHCl} 3$ fraction) from Xylocarpus granatum depicted antimicrobial activity against Staphylococcus epidermis (20-25 mm), Staphylococcus aureus $(20-25 \mathrm{~mm})$, Shigella boydii (20-25 mm), Proteus spp. (20-25 mm), Escherichia coli (20-25 mm), and Streptococcus pyogenes $(20-25 \mathrm{~mm})$ in terms of disc inhibition zone (diameter in $\mathrm{mm}$ ) at concentration of $400 ? \mathrm{~g} / \mathrm{disc}$. However, the exact mechanism is not known [82].

3.5.12. Antidiarrheal Effect. Xylocarpus granatum bark extracts (methanol extracts) when supplemented in mice induced with diarrhea (induced using castor oil and magnesium sulphate) revealed that, at oral dosage of 250 and $500 \mathrm{mg} / \mathrm{kg}$, it exhibited antidiarrheal properties by reducing the wet faeces discharge. Castor oil is known to cause diarrhea due to its hypersecretory activity caused by active component ricinoleic acid, and the antidiarrheal activity of the Xylocarpus granatum bark extracts could be due to its antisecretory effect [83].

These studies, highlighted in the present review on the different pharmacological activities exhibited by parts of Xylocarpus granatum, its extracts, and bioactive compounds, indicate the health promoting effects of Xylocarpus granatum, which thus justifies their application as indigenous medicine in different traditional medicinal system used across the globe. It also paves way for further research to translate these natural plant-based bioactive compounds as advanced pharmaceutical drugs for treating various disorders, thus allowing researchers to explore the best of both scientific as well as traditional knowledge systems.

\section{Toxicity}

Limited research is available on the toxicity of $X$. granatum and its extracts. However, a study depicted that oral dosage of ethanolic bark extract of X. granatum at $1000 \mathrm{mg} / \mathrm{kg}$ body weight per day when given to healthy Balb/c mice showed no signs of toxic effects or death up to four days [81]. Another study indicated that ethyl acetate extract of $X$. granatum leaves had no toxicity when tested using brine shrimp lethality test (BSLT) even above 1500 ppm concentration [62]. In conclusion, though few studies indicate no toxicity, detailed experiments are necessary to further establish the toxicity or upper tolerable limits. 


\section{Conclusion and Future Prospects}

X. granatum may be one of the hopeful medicinal herbs for the treatment of various diseases in humans. Traditionally, this plant was widely used in treating several diseases, because of its chemical constituents. Several studies have shown that $X$. granatum has anticancer, antiviral, antidepressant, antifeedant, and antimalarial activities ascribed to its chemical constituents such as limonoids, phragmalin, limonoid-based alkaloids, mexicanolides, protolimonoids, flavonol-like compounds, and alkaloids, among others. These chemical compounds are essential to treat many diseases and should be established as standard drugs for several known physiological disorders and diseases in medicinal chemistry and pharmacology. Still there is a lack of clinical trials on utilization of $X$. granatum and its bioactive compounds or extracts, and fewer studies are conducted on exploiting the development of plant-based drugs using $X$. granatum. Thus, more research is necessary to understand its chemical compounds and utilization as novel drugs in human studies and investigate the mechanism of action in treating various ailments. Also, the toxicological aspects of this medicinal mangrove plant need to be investigated thoroughly before developing any novel drug as limited studies are available.

\section{Conflicts of Interest}

The authors declare that they have no conflicts of interest.

\section{Acknowledgments}

This work was supported by CONICYT PIA/APOYO CCTE AFB170007.

\section{References}

[1] H. Yuan, Q. Ma, L. Ye, and G. Piao, "The traditional medicine and modern medicine from natural products," Molecules, vol. 21, 2016.

[2] Q. Gao, Y. Gao, H. Song et al., "Cipadesin A, a bioactive ingredient of Xylocarpus granatum, produces antidepressantlike effects in adult mice," Neuroscience Letters, vol. 633, pp. 33-39, 2016.

[3] A. F. M. Shahid-Ud-Daula and M. A. Basher, "Phytochemical screening, plant growth inhibition, and antimicrobial activity studies of Xylocarpus granatum," Malaysian Journal of Pharmaceutical Sciences, vol. 7, pp. 9-21, 2009.

[4] S. K. Das, D. Samantaray, S. K. Sahoo, S. K. Pradhan, L. Samanta, and H. Thatoi, "Bioactivity guided isolation of antidiabetic and antioxidant compound from Xylocarpus granatum J. Koenig bark," 3 Biotech, vol. 9, no. 5, p. 198, 2019.

[5] M. E. Islam, S. M. Rahman, M. H. Sohrab, R. Biswas, M. S. Ullah, and K. D. Islam, "Concordance of antioxidant and anti-Inflammatory activity in Xylocarpus granatum (Koen)," Journal of the Bangladesh Agricultural University, vol. 17, no. 4, pp. 466-475, 2019.

[6] U. Kokpol, W. Chavasiri, S. Tip-Pyang et al., "A limonoid from Xylocarpus granatum," Phytochemistry, vol. 41, no. 3, pp. 903-905, 1996.
[7] F. Cheng, Y. Zhou, K. Zou, and J. Wu, "Studies on the chemical constituents of the fruit of Xylocarpus granatum," Zhong Yao Cai, vol. 32, pp. 1220-1223, 2009.

[8] J. Cui, J. Wu, Z. Deng, P. Proksch, and W. Lin, "Xylocarpins A-I, Limonoids from the Chinese mangrove plant Xylocarpus granatum," Journal of Natural Products, vol. 70, no. 5, pp. 772-778, 2007.

[9] X. Shi, Y. Wu, T. Lv et al., "A chemometric-assisted LC-MS/ MS method for the simultaneous determination of 17 limonoids from different parts of Xylocarpus granatum fruit," Analytical and Bioanalytical Chemistry, vol. 409, no. 19, pp. 4669-4679, 2017.

[10] J. Wu, Q. Xiao, S. Zhang et al., "Xyloccensins Q-V, Six new 8,9,30-phragmalin ortho ester antifeedants from the Chinese mangrove Xylocarpus granatum," Tetrahedron, vol. 61, no. 35, pp. 8382-8389, 2005.

[11] R. Tundis, M. R. Loizzo, and F. Menichini, "An overview on chemical aspects and potential health benefits of limonoids and their derivatives," Critical Reviews in Food Science and Nutrition, vol. 54, no. 2, pp. 225-250, 2014.

[12] V. Lakshmi, S. Srivastava, S. K. Mishra, M. N. Srivastava, K. Srivastava, and S. K. Puri, "Antimalarial activity in Xylocarpus granatum (Koen)," Natural Product Research, vol. 26, no. 11, pp. 1012-1015, 2012.

[13] S. Baba, H. Chan, M. Kainuma, M. Kezuka, E. W. Chan, and J. Tangah, "Botany, uses, chemistry and bioactivities of mangrove plants III: Xylocarpus granatum," ISME/GLOMIS Electronic Journal, vol. 14, pp. 1-4, 2016.

[14] S. Raja and K. Ravindranadh, "A complete profile on Xylocarpus moluccensis: traditional uses, pharmacological activities and phytoconstituents," World Journal of Pharmaceutical Sciences, vol. 2, pp. 1770-1777, 2014.

[15] B. Yin, C. Huo, L. Shen et al., "Protolimonoids from the seeds of Xylocarpus granatum," Biochemical Systematics and Ecology, vol. 37, no. 3, pp. 218-220, 2009.

[16] S. J. Uddin, L. Nahar, J. A. Shilpi et al., "Gedunin, a limonoid from Xylocarpus granatum, inhibits the growth of CaCo-2 colon cancer cell line in vitro," Phytotherapy Research, vol. 21, no. 8, pp. 757-761, 2007.

[17] M. Ross, A. K. Pawley, and M. Osmond, The Lexicon of ProtoOceanic: the Culture and Environment of Ancestral Oceanic Society, Part 3: PlantsAustralian National University, Canberra, Australia, 2008.

[18] J. S. Bunt, W. T. Williams, and N. C. Duke, "Mangrove distributions in north-east Australia," Journal of Biogeography, vol. 9, no. 2, pp. 111-120, 1982.

[19] O. C. Steele, Natural and Anthropogenic Biogeography of Mangroves in the Southwest Pacific, University of Hawaii at Manoa, Honolulu, Hawaii, 2006.

[20] V. Lakshmi and P. Gupta, "An overview of the genus Xylocarpus," Natural Product Research, vol. 22, no. 14, pp. 1197-1224, 2008.

[21] B. Sarkar, M. A. Ullah, M. S. Islam, S. Hossain, and M. NafiUr-Rahman, "Anticancer potential of medicinal plants from Bangladesh and their effective compounds against cancer," Journal of Pharmacognosy and Phytochemistry, vol. 8, pp. 827-833, 2019.

[22] S. Du, M. Wang, W. Zhu, and Z. Qin, "A new fungicidal lactone from Xylocarpus granatum (Meliaceae)," Natural Product Research, vol. 23, no. 14, pp. 1316-1321, 2009.

[23] H. Wangensteen, G. M. Duong, M. Alamgir, M. Sarder, A. B. Samuelsen, and K. E. Malterud, "Biological activities of limonoids, catechins, procyanidins and extracts from 
Xylocarpus granatum," Natural Product Communications, vol. 1, no. 11, Article ID 1934578X0600101, 2006.

[24] A. S. Ng and A. G. Fallis, "Comment: $7 \alpha$-Acetoxydihydronomilin and mexicanolide: limonoids from Xylocarpus granatum (Koenig)," Canadian Journal of Chemistry, vol. 57, no. 23, pp. 3088-3089, 1979.

[25] K. Pudhom, D. Sommit, P. Nuclear, N. Ngamrojanavanich, A. Petsom, and F.-H. Protoxylocarpins, "Protolimonoids from seed kernels of Xylocarpus granatum," Journal of Natural Products, vol. 72, no. 12, pp. 2188-2191, 2009.

[26] K. Toume, K. Kamiya, M. A. Arai et al., "Xylogranin B: a potent Wnt signal inhibitory limonoid from Xylocarpus granatum," Organic Letters, vol. 15, no. 23, pp. 6106-6109, 2013.

[27] Y.-G. Dai, J. Wu, K. P. Padmakumar, and L. Shen, "Sundarbanxylogranins A-E, five new limonoids from the Sundarban Mangrove, Xylocarpus granatum," Fitoterapia, vol. 122, pp. 85-89, 2017.

[28] R.-X. Liu, Q. Liao, L. Shen, and J. Wu, "Krishnagranatins A-I: new limonoids from the mangrove, Xylocarpus granatum, and NF- $\kappa$ B inhibitory activity," Fitoterapia, vol. 131, pp. 96-104, 2018.

[29] M. Liao, P. Pedpradab, and J. Wu, "Thaixylogranins A-H: eight new limonoids from the Thai mangrove, Xylocarpus granatum," Phytochemistry Letters, vol. 19, pp. 126-131, 2017.

[30] S. Yin, X.-N. Wang, C.-Q. Fan, L.-P. Lin, J. Ding, and J.-M. Yue, "Limonoids from the seeds of the marine Mangrove Xylocarpus granatum," Journal of Natural Products, vol. 70, no. 4, pp. 682-685, 2007.

[31] C.-H. Huo, D. Guo, L.-R. Shen et al., "Xylocarpanoids A and B, unique C28 skeleton limonoids from Xylocarpus granatum," Tetrahedron Letters, vol. 51, no. 4, pp. 754-757, 2010.

[32] L.-R. Shen, M. Dong, B.-W. Yin et al., "Xylomexicanins A and B, new d14,15-mexicanolides from seeds of the Chinese mangrove Xylocarpus granatum," Zeitschrift für Naturforschung C, vol. 64, no. 1-2, pp. 37-42, 2009.

[33] Y.-B. Wu, Z.-Y. Ni, C.-H. Huo et al., "Xylomexicanins C and $\mathrm{D}$, new mexicanolide-type limonoids from Xylocarpus granatum," Bioscience, Biotechnology, and Biochemistry, vol. 77, no. 4, pp. 736-740, 2013.

[34] Y.-B. Wu, Y.-Z. Wang, Z.-Y. Ni et al., "Xylomexicanins I and $\mathrm{J}$ : limonoids with unusual $\mathrm{B} / \mathrm{C}$ rings from Xylocarpus granatum," Journal of Natural Products, vol. 80, no. 9, pp. 2547-2550, 2017.

[35] J. Cui, J. Ouyang, Z. Deng, and W. Lin, "Structure elucidation of an unprecedented alkaloid and a new limonoid from Xylocarpus granatum," Magnetic Resonance in Chemistry, vol. 46, no. 9, pp. 894-897, 2008.

[36] Z.-F. Zhou, L.-Y. Kong, T. Kurtán et al., "Four phragmalin orthoesters from the Chinese mangrove Xylocarpus granatum," Planta Medica, vol. 80, no. 11, pp. 949-954, 2014.

[37] J.-Y. Pan, S.-L. Chen, M.-Y. Li, J. Li, M.-H. Yang, and J. Wu, "Limonoids from the seeds of a hainan mangrove, Xylocarpus granatum," Journal of Natural Products, vol. 73, no. 10, pp. 1672-1679, 2010.

[38] J. Wu, S. Zhang, T. Bruhn, Q. Xiao, H. Ding, and G. Bringmann, "Xylogranatins F-R: antifeedants from the Chinese Mangrove, Xylocarpus granatum, A new biogenetic pathway to tetranortriterpenoids," Chemistry - A European Journal, vol. 14, no. 4, pp. 1129-1144, 2008.

[39] J.-L. Ren, X.-P. Zou, W.-S. Li, L. Shen, and J. Wu, "Limonoids containing a C1-O-C29 moiety: isolation, structural modification, and antiviral activity," Marine Drugs, vol. 16, no. 11, p. 434, 2018.
[40] Y. Wu, Y. Bai, X. Guo et al., “A new limonoid from Xylocarpus granatum," Chemistry of Natural Compounds, vol. 50, no. 2, pp. 314-316, 2014.

[41] J. Li, M.-Y. Li, T. Bruhn et al., "Andhraxylocarpins A-E: structurally intriguing limonoids from the true Mangrove Xylocarpus granatum and Xylocarpus moluccensis," ChemistryA European Journal, vol. 18, no. 45, pp. 14342-14351, 2012.

[42] W.-M. Hu and J. Wu, "Protoxylogranatin B, a key biosynthetic intermediate from Xylocarpus granatum: suggesting an oxidative cleavage biogenetic pathway to limonoid," The Open Natural Products Journal, vol. 3, no. 1, pp. 1-5, 2010.

[43] M.-Y. Li, J. Wu, S. Zhang, Q. Xiao, and Q.-X. Li, “The absolute stereochemistry of protoxylogranatin A - a new protolimonoid from the seeds of Chinese mangrove Xylocarpus granatum," Journal of Asian Natural Products Research, vol. 10, no. 6, pp. 503-508, 2008.

[44] J. Wu, S. Zhang, Q. Xiao et al., "Xyloccensin M and N, two new B, D-seco limonoids from Xylocarpus granatum," Zeitschrift für Naturforschung B, vol. 58, no. 12, pp. 1216-1219, 2003.

[45] J. Wu, S. Zhang, Q. Xiao et al., "Xyloccensin L, a novel limonoid from Xylocarpus granatum," Tetrahedron Letters, vol. 45, no. 3, pp. 591-593, 2004.

[46] K. A. Alvi, P. Crews, B. Aalbersberg, and R. Prasad, "Limonoids from the Fijian medicinal plant dabi (xylocarpus)," Tetrahedron, vol. 47, no. 43, pp. 8943-8948, 1991.

[47] J. Wu, Q. Xiao, J. Huang et al., "Xyloccensins $\mathrm{O}$ and $\mathrm{P}$, unique 8,9,30-phragmalin ortho esters from Xylocarpus granatum," Organic Letters, vol. 6, no. 11, pp. 1841-1844, 2004.

[48] V. Lakshmi, N. Singh, S. Shrivastva et al., "Gedunin and photogedunin of Xylocarpus granatum show significant antisecretory effects and protect the gastric mucosa of peptic ulcer in rats," Phytomedicine, vol. 17, no. 8-9, pp. 569-574, 2010.

[49] F. Cheng, Y. Zhou, J. Wu, and K. Zou, "Xyloccensins X1 and $\mathrm{X} 2$, two new mexicanolides from the fruit of a Chinese mangrove Xylocarpus granatum," Zeitschrift für Naturforschung B, vol. 61, no. 5, pp. 626-628, 2006.

[50] J. Wu, H. Ding, M. Li, and S. Zhang, "Xylogranatin E, a new phragmalin with a rare oxygen bridge between C1 and C29, from the fruit of a Chinese mangrove Xylocarpus granatum," Zeitschrift für Naturforschung B, vol. 62, no. 4, pp. 569-572, 2007.

[51] Y. Zhou, J. Wu, and K. Zou, "Xylogranatinin, a new pyrido[1, 2-a] pyrazine alkaloid from the fruit of a Chinese mangrove Xylocarpus granatum," Chemistry of Natural Compounds, vol. 43, no. 4, pp. 426-428, 2007.

[52] Z.-F. Zhou, H.-L. Liu, W. Zhang et al., "Bioactive rearranged limonoids from the Chinese mangrove Xylocarpus granatum Koenig," Tetrahedron, vol. 70, no. 37, pp. 6444-6449, 2014.

[53] Z.-F. Zhou, O. Taglialatela-Scafati, H.-L. Liu, Y.-C. Gu, L.-Y. Kong, and Y.-W. Guo, "Apotirucallane protolimonoids from the Chinese mangrove Xylocarpus granatum Koenig," Fitoterapia, vol. 97, pp. 192-197, 2014.

[54] J. Wu, Q. Xiao, and Q. Li, "Limonoids from the mangrove Xylocarpus granatum," Biochemical Systematics and Ecology, vol. 34, no. 11, pp. 838-841, 2006.

[55] J. C. Zhang, Q. Liao, L. Shen, and J. Wu, "Twenty-five limonoids from the Hainan mangrove, Xylocarpus granatum," Bioorganic Chemistry, vol. 100, Article ID 103903, 2020.

[56] S. Misra, M. Verma, S. K. Mishra, S. Srivastava, V. Lakshmi, and S. Misra-Bhattacharya, "Gedunin and photogedunin of Xylocarpus granatum possess antifilarial activity against human lymphatic filarial parasite Brugia malayi in experimental 
rodent host," Parasitology Research, vol. 109, no. 5, pp. 1351-1360, 2011.

[57] Y. S. Sang, C. Y. Zhou, A. J. Lu, X. J. Yin, Z. D. Min, and R. X. Tan, "Protolimonoids from melia toosendan," Journal of Natural Products, vol. 72, no. 5, pp. 917-920, 2009.

[58] W. Terra, I. Vieira, R. Braz-Filho, W. Freitas, M. Kanashiro, and $\mathrm{M}$. Torres, "Lepidotrichilins $\mathrm{A}$ and $\mathrm{B}$, new protolimonoids with cytotoxic activity from Trichilia lepidota (Meliaceae)," Molecules, vol. 18, no. 10, pp. 12180-12191, 2013.

[59] J. K. R. R. Samarasekera, B. P. S. Khambay, and K. P. Hemalal, "A new insecticidal protolimonoid from Aegle marmelos," Natural Product Research, vol. 18, no. 2, pp. 117-122, 2004.

[60] J.-R. Wang, H.-L. Liu, T. Kurtán et al., "Protolimonoids and norlimonoids from the stem bark of Toona ciliata var. pubescens," Organic and Biomolecular Chemistry, vol. 9, no. 22, pp. 7685-7696, 2011.

[61] Y.-G. Dai, W.-S. Li, P. Pedpradab, J.-J. Liu, J. Wu, and L. Shen, "Thaixylomolins O-R: four new limonoids from the Trang mangrove, Xylocarpus moluccensis," RSC Advances, vol. 6, no. 89, pp. 85978-85984, 2016.

[62] J. Darmadi, R. R. Batubara, S Himawan et al., "Evaluation of Indonesian mangrove Xylocarpus granatum leaves ethyl acetate extract as potential anticancer drug," Scientific Reports, vol. 11, no. 1, pp. 6080-6118, 2021.

[63] WHO, Report of the WHO Informal Consultation on the "Evaluation and Testing of Insecticides", World Health Organization (WHO), Geneva, Switzerland, 1996.

[64] S. Mohammadi, B. Jafari, P. Asgharian, M. Martorell, and J. Sharifi-Rad, "Medicinal plants used in the treatment of Malaria: a key emphasis to Artemisia, Cinchona, Cryptolepis, and Tabebuia genera," Phytotherapy Research, vol. 34, no. 7, pp. 1556-1569, 2020.

[65] S. A. Khalid, H. Duddeck, and M. Gonzalez-Sierra, "Isolation and characterization of an antimalarial agent of the neem tree Azadirachta indica," Journal of Natural Products, vol. 52, no. 5, pp. 922-927, 1989.

[66] L. M. Schoonhoven, "Biological aspects of antifeedants," Entomologia Experimentalis et Applicata, vol. 31, no. 1, pp. 57-69, 1982.

[67] J. Cui, Z. Deng, J. Li, H. Fu, P. Proksch, and W. Lin, "Phragmalin-type limonoids from the mangrove plant Xylocarpus granatum," Phytochemistry, vol. 66, no. 19, pp. 2334-2339, 2005.

[68] J. S. Flier, L. H. Underhill, and G. S. Eisenbarth, "Type I diabetes mellitus," New England Journal of Medicine, vol. 314, no. 21, pp. 1360-1368, 1986.

[69] M. A. Atkinson, G. S. Eisenbarth, and A. W. Michels, "Type 1 diabetes," The Lancet, vol. 383, no. 9911, pp. 69-82, 2014.

[70] S. P. Srivastava, A. Mishra, V. Lakshmi, A. K. Tamrakar, M. N. Srivastava, and A. K. Srivastava, "Antidiabetic and antidyslipidemic activity of ethyl acetate fractions of Xylocarpus granatum and Xylocarpus molluccensis on high fructose high fat and high sucrose high fat fed-low dosed streptozotocin treated diabetic rats," International Journal of Pharmacy and Pharmaceutical Sciences, vol. 7, pp. 537-543, 2015.

[71] M. Kumar, M. Tomar, R. Amarowicz et al., "Guava (Psidium guajava L.) leaves: nutritional composition, phytochemical profile, and health-promoting bioactivities," Foods, vol. 10, no. 4, p. 752, 2021.

[72] M. Kumar, V. Saurabh, M. Tomar et al., "Mango (Mangifera indica L.) leaves: nutritional composition, phytochemical profile, and health-promoting bioactivities," Antioxidants, vol. 10, no. 2, p. 299, 2021.
[73] S. Punia and M. Kumar, "Litchi (Litchi chinenis) seed: nutritional profile, bioactivities, and its industrial applications," Trends in Food Science \& Technology, 2020.

[74] C. S. Mackenzie, K. Reynolds, K.-L. Chou, J. Pagura, and J. Sareen, "Prevalence and correlates of generalized anxiety disorder in a national sample of older adults," American Journal of Geriatric Psychiatry, vol. 19, no. 4, pp. 305-315, 2011.

[75] M. Prince, V. Patel, S. Saxena et al., "No health without mental health,” The Lancet, vol. 370, no. 9590, pp. 859-877, 2007.

[76] S. R. M. Kadali, A. S. R. S. Rao, and G. K. Sri, "Antidepressant activity of brahmi in albino mice," Journal of Clinical and Diagnostic Research, vol. 8, pp. 35-37, 2014.

[77] A. Markou and J. F. Cryan, "Stress, anxiety and depression: toward new treatment strategies," Neuropharmacology, vol. 62, no. 1, pp. 1-2, 2012.

[78] M. C. Castro, S. Kanamori, K. Kannady, S. Mkude, G. F. Killeen, and U. Fillinger, "The importance of drains for the larval development of lymphatic filariasis and malaria vectors in Dar es Salaam, United Republic of Tanzania," PLoS Neglected Tropical Diseases, vol. 4, no. 5, p. e693, 2010.

[79] L. A. Lacey and A. H. Undeen, "Microbial control of black flies and mosquitoes," Annual Review of Entomology, vol. 31, no. 1, pp. 265-296, 1986.

[80] N. Robbins, G. D. Wright, and L. E. Cowen, "Antifungal drugs: the current armamentarium and development of new agents," Microbiology Spectrum, vol. 4, no. 5, 2016.

[81] S. K. Das, A. Prusty, D Samantaray et al., "Effect of Xylocarpus granatum bark extract on amelioration of hyperglycaemia and oxidative stress associated complications in STZ-induced diabetic mice," Evidence-based Complementary and Alternative Medicine: eCAM, vol. 2019, Article ID 8493190, 13 pages, 2019.

[82] M. Alam, M. Sarder, M. Awal, M. Sikder, and K. Daulla, "Antibacterial activity of the crude ethanolic extract of Xylocarpus granatum stem barks," Bangladesh Journal of Veterinary Medicine, vol. 4, pp. 69-72, 2006.

[83] R. Rouf, S. J. Uddin, J. A. Shilpi, and M. Alamgir, “Assessment of antidiarrhoeal activity of the methanol extract of Xylocarpus granatum bark in mice model," Journal of Ethnopharmacology, vol. 109, no. 3, pp. 539-542, 2007. 